http://economix.fr

\title{
Rueff, Allais, et le chômage d'équilibre
}

Document de Travail

Working Paper

2015-30

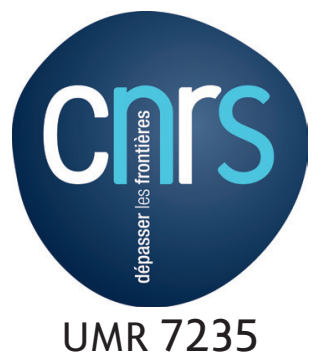

Université de Paris Ouest Nanterre La Défense (bâtiment G) 


\title{
Rueff, Allais, et le chômage d'équilibre
}

\section{Georges Prat}

\author{
Novembre 2015 \\ IPAG Business School (Paris) et EconomiX (CNRS \\ $\&$ Université de Paris Ouest - Nanterre la Défense, France). \\ E-mail : georges.prat@u-paris10.fr
}

\begin{abstract}
Résumé - Le concept de chômage «permanent» proposé par Rueff $(1925,1931)$ représente une forme classique de chômage attribuable à des rigidités salariales conduisant à un excès des salaires réels par rapport à leur valeur théorique correspondant à un équilibre concurrentiel. Par rapport à cet aspect connu des travaux de l'auteur sur le sujet, cet article montre que Rueff considérait aussi la possibilité d'un chômage «temporaire » dû à un niveau insuffisant de l'activité économique et d'un chômage «minimal» de type frictionnel prévalant dans le fonctionnement normal de toute économie. Sur la base d'une étude empirique, l'auteur soutenait que le chômage "permanent» constituait la cause majeure du chômage dans l'Angleterre des années 1920 («loi de Rueff»). Nous présentons des tests économétriques simples sur les données utilisées par Rueff confirmant cette conclusion, tout en montrant que le relâchement de l'hypothèse d'une productivité du travail constante (admise par Rueff) permet une amélioration de la «loi de Rueff». Dans le droit fil de ces résultats, nous montrons que l'analyse théorique d'Allais (1943) repose sur les trois types de chômage évoqués par Rueff, qualifiés désormais de «chronique », «conjoncturel» et «technologique ». Bien plus tard, Allais (1980) a proposé une équation économétrique méconnue intégrant ces trois composantes du chômage en montrant que cette dernière décrit l'évolution du chômage français au cours des années 1952-78. Nous montons que cette équation peut aussi représenter les grands traits des évolutions du chômage anglais des années 1920 et du chômage français des années 1970-2008, mais aussi qu'elle présente un défaut de spécification. Enfin, sous certaines conditions restrictives, nous suggérons que les trois types de chômage distingués par Rueff et Allais peuvent être vus sous le prisme du taux de chômage d'équilibre déduit d'une version simple du modèle de concurrence imparfaite WS-PS (Layard-Nickel-Jackman (1991)), cet exercice indiquant des raisons plausibles au défaut de spécification constatés sur l'équation simple d'Allais, mettant ainsi en évidence les importants progrès scientifiques accomplis depuis.
\end{abstract}

Mots clefs : chômage d'équilibre, rigidité de salaire, Maurice Allais, Jacques Rueff

\begin{abstract}
Rueff, Allais, and the equilibrium unemployment. The «permanent » unemployment concept of Rueff $(1925,1931)$ represents a classical form of unemployment due to wage rigidities leading to an excess of real wages compared to their theoretical value corresponding to a competitive equilibrium. Relative to this known aspect of the Rueff's work, this paper shows that the author considered also a "temporary" unemployment due to an insufficient level of the economic activity, and a «minimum » frictional unemployment prevailing in the normal functioning of any economy. Using empirical data in England during the 1920's, Rueff suggested that the «permanent» component was the main explanation of the unemployment (this is the socalled «law of Rueff»). We conduct simple econometric tests using Rueff's data that confirm this conclusion, and we show that releasing the assumption of a constant labor productivity (supposed by Rueff) improves the «law of Rueff». In line with these results, we show that the theoretical approach proposed by Allais (1943) joins the three types of unemployment pointed out by Rueff, renamed as "chronic", "conjonctural" and "technological", respectively. Much later, Allais (1980) proposed an unrecognized straightforward econometrical equation comprising these three types of unemployment to represent the french unemployment over the period 1952-78. We confirm that this equation describes a large part of the evolution of unemployment in England during the 1920's and in France during the period 1970-2008, although the properties of residuals show that this equation is misspecified. Finally, we suggest that, under some restrictive conditions, the three types of unemployment distinguished by Rueff and Allais can be seen from the perspective of the equilibrium unemployment defined by the imperfect competition WS-PS model (Layard-Nickel-Jackman (1991)), hence allowing to indicate how the Allais' equation is misspecified, thus highlighting the important scientific advances that have been made since.
\end{abstract}

Keywords : equilibrium unemployment, wage rigidities, Maurice Allais, Jacques Rueff

Classification J.E.L. : E24, J2, N34 


\section{Rueff, Allais, et le chômage d'équilibre}

\section{Introduction}

Le concept de chômage «permanent» de Jacques Rueff (1896-1978) représente une forme classique de chômage d'équilibre attribuable à une rigidité structurelle à la baisse des salaires nominaux ayant pour effet de maintenir les salaires réels au dessus de leur niveau d'équilibre concurrentiel. ${ }^{1}$ Par rapport à cet aspect connu des travaux de l'auteur sur le sujet, cet article a trois principaux objectifs qui sont liés. Le premier est de montrer que, contrairement à ce qui est généralement admis, la conception de Rueff dépasse celle du seul chômage «permanent» car elle intègre aussi la possibilité de facteurs conjoncturels et frictionnels du sous-emploi. Le second objectif de cet article est alors de montrer combien l'approche très peu connue proposée par Maurice Allais (1911-2010) rejoint les trois sources du chômage distinguées par Rueff. Partant de ces constats sur les contributions de Rueff et d'Allais, un troisième objectif est de montrer que, sous certaines conditions, les analyses de ces deux auteurs peuvent être vues à travers le prisme du concept moderne de chômage d'équilibre tel qu'il a été défini dans cadre du modèle de concurrence imparfaite WS-PS, ce dernier décrivant les comportements microéconomiques sous-jacents aux facteurs macroéconomiques du chômage (Layard-Nickel-Jackman (1991)). Ce nouveau regard permettra non seulement d'interpréter les approches de Rueff et d'Allais sur la base des comportements microéconomiques explicites des salariés et des employeurs, mais encore mettra en évidence les raisons pour lesquelles les visions de ces deux auteurs étaient trop simples pour rendre compte du chômage d'une manière satisfaisante, mettant ainsi en évidence les importants progrès scientifiques qui ont été accomplis depuis.

A ces fins, cet article est organisé en trois parties. La Partie 1 présente la contribution de Rueff $(1925,1931)$ sur le chômage anglais des années 1920 en l'accompagnant des nombreux débats qu'elle a suscités à l'époque quant à son interprétation. Nous montrerons ce qui à notre connaissance n'a jamais été fait jusqu'ici - que l'auteur distinguait sur le plan théorique trois types de chômage : un chômage «permanent» attribuable à un excès des salaires réels par rapport au rendement du travail («loi de Rueff»), un chômage «temporaire » attribuable à un niveau insuffisant de l'activité économique provoqué par une diminution cyclique des prix, et enfin un chômage «minimal » de type frictionnel prévalant dans le fonctionnement normal de toute économie. Sur la base d'observations empiriques portant sur l'Angleterre des années 1919-30, l'auteur soutenait que le chômage "permanent » constituait la cause majeure du chômage, ceci en supposant constante la productivité du travail représentant le salaire réel d'équilibre concurrentiel ; en remettant en cause cette hypothèse, nous montrons que, loin d'être infirmée, la «loi de Rueff» devient encore plus précise. Par ailleurs, Rueff supposait que le chômage «temporaire » pouvait être négligé ; nous montrons à ce propos que si cette conjecture s'avère vérifiée à partir de 1924, elle ne semble pas valide en 1919-21 ainsi qu'en 1930, à un moindre degré. Enfin, nous présenterons en tant que besoin des tests économétriques simples dits de «causalité » sur les données utilisées par Rueff, ces tests étant destinés à éclairer les diverses interprétations envisagées

\footnotetext{
${ }^{1}$ Cet article a été rédigé à la suite de notre communication présentée au colloque sur Jacques Rueff à Paris le 23 mai 2013 organisé conjointement par l'IPAG Business School (Paris), l'université de Clermont-Ferrand et 1'Ecole Polytechnique de Paris (Prat, 2013).
} 
dans la littérature de l'époque, les résultats obtenus confortant dans l'ensemble les idées de Rueff.

Dans le droit fil de ces résultats, la Partie 2 montre qu'Allais a confirmé en les précisant les idées de Rueff sur les causes du chômage, sans toutefois marquer combien son approche se décalque sur celle de ce dernier. En effet, suite à l'exposé théorique présenté dans son Traité de 1943, Allais a proposé beaucoup plus tard une équation empirique simple intégrant les trois types de chômage distingués par Rueff qu'il désigne respectivement par les qualificatifs de «chronique », «conjoncturel » et «technologique ». En outre, Allais a montré que son équation décrit l'évolution du chômage français sur la période 1952-78 (Allais, 1980), mais n'ayant pas été publiés dans des supports académiques, ces résultats sont restés très peu connus. Afin d'en apprécier la robustesse, nous présentons des estimations de cette équation pour l'Angleterre des années 1920 et pour la France sur la période 1970-2008; si les résultats obtenus tendent à confirmer la pertinence des trois sources de chômage distingués par Rueff et Allais, par contre, l'analyse des résidus montrent un défaut de spécification de cette équation, ce qui n'est guère très étonnant compte-tenu de sa grande simplicité.

Bien que Rueff et Allais aient largement affiché leurs divergences avec les idées de Keynes, la nouvelle économie keynésienne (NEK) des années 1975-90 a approfondi l'analyse des facteurs de rigidité des salaires, pierre d'achoppement tant de la «loi de Rueff» que de l'approche d'Allais. Cependant, alors que ces deux auteurs voyaient des causes exogènes liées aux règlementations sociales mises en place et imposées par l'Etat (allocations versées aux chômeurs; cotisations sociales obligatoires alourdissant les charges salariales), la NEK montre que la rigidité des salaires peut aussi être le résultat des comportements endogènes des salariés et des entreprises. Le modèle de concurrence imparfaite WS-PS proposé par LayardNickel-Jackman (1990) se situe dans la lignée de cette littérature en explicitant les comportements de négociation des salariés et des employeurs tout en prenant en compte les causes exogènes de rigidité. L'objectif de la Partie 3 est de montrer que, sous certaines conditions restrictives, on peut formellement réinterpréter les trois composantes du chômage de Rueff et d'Allais au regard du taux de chômage d'équilibre défini dans le cadre du modèle WS-PS où les comportements microéconomiques sous-jacents sont explicités. Cet exercice contribuera ainsi à éclairer les raisons possibles du défaut de spécification que nous avons pu constater concernant l'équation d'Allais. En conclusion, nous dégagerons les principaux enseignements des analyses et des rapprochements effectués tout au long de cet article.

\section{1 - Les facteurs du chômage dans l'Angleterre des années 1920 selon Rueff : faits, interprétations et débats}

Rueff fut à son époque un économiste très écouté, lu et respecté, mais aussi souvent controversé, notamment concernant ses contributions sur les questions monétaires internationales et la balance des paiements. ${ }^{2}$ Sa reconnaissance posthume reste néanmoins relativement limitée à ce jour dans le monde académique. Nous montrons ici que son apport sur le sujet particulier du chômage mérite une attention sans doute plus grande que celle qui lui est accordée. Au plan de la pensée économique en général, Rueff disait lui-même avoir hérité de l'enseignement de Clément Colson (1853-1939) ainsi que de la lecture des œuvres de Léon Walras (1834-1910) et de Charles Rist (1874-1955), lesquels faisaient confiance au

\footnotetext{
${ }^{2}$ Voir l'autobiographie de Rueff (1977) ainsi que Teulon (2014).
} 
mécanisme du marché, même s'ils pensaient que l'intervention de l'Etat peut se justifier pour faire respecter les règles du jeu concurrentiel ou pour des raisons sociales. Sur la question précise du chômage, il est très vraisemblable que Rueff ait été influencé par Arthur Cecil Pigou (1877-1959) qui fut un élève d'Alfred Marshall (1845-1924), ardent défenseur du «laisser faire, laisser passer ». Par ailleurs, dans sa démarche, Rueff se souciait de mettre en cohérence les faits observés avec l'analyse économique et, en ce sens, il a fait preuve d'une grande lucidité pour éclairer son époque. En particulier, dans ses articles de 1925 et $1931^{3}$, il a confirmé empiriquement pour l'Angleterre des années 1920 un corollaire de la théorie classique suivant lequel, si le salaire réel se maintient durablement au-dessus de sa valeur correspondant à un équilibre concurrentiel, cette situation produit du chômage "permanent », phénomène souvent appelé «loi de Rueff» par les commentateurs.

\subsection{La thèse d'un chômage «permanent » : la «loi de Rueff »}

Au sortir de la première Guerre, dans un contexte inflationniste, la volonté de rétablir la parité-or de la livre-sterling d'avant-guerre avait conduit le Gouvernement britannique à une politique déflationniste. Cette politique a réussi puisque les prix de gros ont été divisés par environ 3 entre la mi-1920 et la fin de 1930. Au cours de la même période, le salaire moyen ${ }^{4}$ ayant été divisé par 1.5, il en résulte que le salaire réel a été multiplié environ par 2, ce qui est considérable. La figure 1 donne les évolutions comparées des indices des prix de gros ${ }^{5}$ et des salaires entre le début de 1919 et la fin de 1930 (données trimestrielles). Nous avons élaboré cette figure à partir des données fournies par Rueff dans l'Annexe I de son article de 1931. ${ }^{6}$ A partir de 1920, on remarque que la baisse des salaires a été moins rapide que celle des prix ; à partir de 1924, les salaires monétaires sont quasi-stables bien qu'accompagnés d'une baisse des prix. D'après Rueff (1925), «tout se passe comme si le phénomène moteur était la variation de prix, suivie tardivement seulement par une variation de salaire » : la causalité irait donc des prix vers les salaires et non l'inverse. ${ }^{7}$ Comme nous le verrons ci-après avec l'analyse de Pigou, les variations du niveau des prix ont elles-mêmes pour principale source la politique monétaire britannique déflationniste de l'époque.

\footnotetext{
${ }^{3}$ Nous nous référons dans cette partie à deux articles, l'un très court de 1925 publié dans la Revue Politique et Parlementaire et l'autre beaucoup plus étendu publié en 1931dans la Revue d'Économie Politique. Ces articles ont été réédités dans les «Oeuvres complètes de Jacques Rueff», E.M Claassen éd., Tome II, Théorie Monétaire, livre 2, Plon, Paris, p.219-230 et 231-270. Durant les années 1920, Rueff fut inspecteur des finances, puis chargé de mission auprès de Raymond Poincaré et attaché financier à l'ambassade de France à Londres. En raison du rôle éminent qu'il a tenu dans l'Administration française, mais aussi du caractère «brûlant » du sujet et des réactions suscitées par son article de 1925, 1'article de 1931 avait été publié anonymement.

${ }^{4}$ Rueff retient «l'indice général des prix du travail » établi par le professeur Bowley. Dans la grande majorité des cas, les salaires correspondent à la rémunération d'une semaine de travail. Bowley a considéré onze groupes appartenant à des professions distinctes et provenant de diverses régions, les informations de base ayant été collectées dans les publications du ministère du travail anglais. Les cotisations (cotisations sociales et assurance chômage) sont payées par les salariés en \% de leurs salaires.

${ }^{5}$ Indice du Board of Trade. Rueff justifie l'utilisation des prix de gros et non de l'indice du coût de la vie par le fait qu'il cherche plus à mesurer le rapport entre les salaires payés par les entreprises et les prix de vente qu'à rendre compte du pouvoir d'achat des ouvriers. Toutefois, Pigou (1927) lève toute ambiguïté sur ce point en montrant la robustesse des résultats de Rueff lorsqu'un indice général des prix est considéré.

${ }^{6}$ Rueff avait présenté des graphiques en traitant les changements de base au $4{ }^{\text {ème }}$ trimestre 1928 par des glissements d'échelles; nous avons raccordé les séries qui sont désormais exprimées en indices base 1913=100 sur l'ensemble de la période ; en outre, les logarithmes de ces indices sont considérés alors que Rueff considérait les indices bruts.

${ }^{7}$ Cette idée a été reprise avec force dans son article de 1976 : "C'est une erreur et un mensonge d'attribuer à la revendication salariale, fondée sur des exigences idéologiques, la hausse des prix et l'inflation. La revendication salariale ne tend qu'à protéger les niveaux de vie des travailleurs contre les prélèvements occultes dont ils n'ont cessé d'être l'objet. Elle est effet et non pas cause de la hausse des prix».
} 
Figure 1 - Salaires et prix de gros

Angleterre, 1919 - 1930

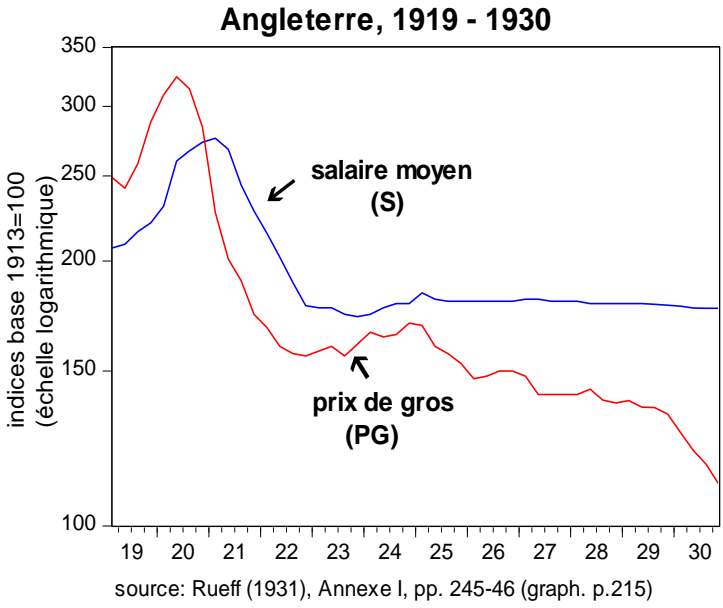

Figure 2 - Taux de chômage et salaire réel Angleterre, $1919-1930$

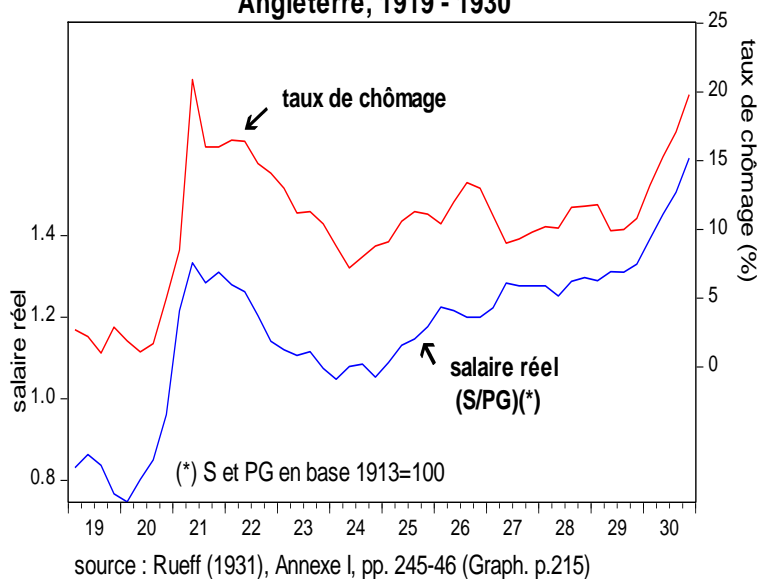

Nous avons appliqué le test de causalité de Granger pour vérifier l'hypothèse de Rueff suivant laquelle ce sont les variations de prix et non celles des salaires qui sont le phénomène moteur. ${ }^{8}$ On doit préciser ici que ce test se distingue de l'argument d'une simple avance temporelle : les prix peuvent a priori précéder les salaires sans pour autant impliquer qu'ils sont une «cause» des salaires au sens de ce test. Les résultats donnés sur la table 1 confirment l'analyse de Rueff, puisque la «p-value » est nulle pour l'hypothèse « les prix ne causent pas les salaires » tandis que la «p-value » correspondant à l'hypothèse «les salaires ne causent pas les prix » dépasse très largement le seuil critique de 5\%. D'autre part, en régressant le taux de variation du salaire moyen sur sa valeur du trimestre précédent et sur le taux de variation des prix, le coefficient autorégressif permettant de chiffrer le délai moyen d'influence de l'inflation sur les variations de salaire à 2.2 trimestres. ${ }^{9}$

Table 1 - Analyse de la causalité au sens de Granger entre les prix de gros $(P G)$ et les salaires (S) - Angleterre : 1919.Q1-1930.Q4

\begin{tabular}{|cccc|}
\hline Null Hypothesis (2 lags) : & N.obs & F-Statistic & p-value \\
& & & \\
$d(\log (P G))$ does not Granger Cause $d(\log (S))$ & 45 & 19.3541 & 1. E-06 \\
$d(\log (S))$ does not Granger Cause $d(\log (P G))$ & & 1.05280 & 0.3584 \\
\hline
\end{tabular}

Notes table $1-d \log \left(X_{t}\right)=\log X_{t}-\log X_{t-1} . S_{t}$ : salaire nominal ; $P G_{t}$ : prix de gros. Les résultats présentés sont établis avec des retards allant jusqu'à à deux trimestres. Nous avons pu vérifier que des retards allant de 1 à 8 trimestres mènent aux mêmes conclusions

Par ailleurs, Rueff (1925) observe qu'entre août 1920 et juin 1921, le nombre des chômeurs augmente de 120.000 à 2.170 .000 : ce nombre a été multiplié par 18 en l'espace de 10 mois seulement ; le taux de chômage a quant à lui augmenté de $1 \%$ au $3^{\text {ème }}$ trimestre 1920 à plus de $20 \%$ au second trimestre 1921 . Le choc a donc été d'une violence extrême et ceci

\footnotetext{
${ }^{8}$ Nous avons préalablement vérifié que les séries sont stationnaires en taux de variation (test ADF). Suivant ce test, les prix (resp. les salaires) sont considérés comme une «cause » des salaires (resp. des prix) au sens de Granger si les variations passées des prix (resp. des salaires) améliorent d'une manière significative la représentation des variations de salaire (resp. des prix) par rapport aux seules valeurs passées de ces dernières. Soulignons ici que la mise en évidence d'une causalité au sens statistique ne dit bien sûr rien en elle-même sur l'explication économique sous-jacente.

9 On obtient : $d \log \left(S_{t}\right)=\underset{(7.0)}{0.69} d \log \left(S_{t-1}\right)+\underset{(3.0)}{0.18} d \log \left(P G_{t}\right)+\underset{(0.6)}{0.002} \quad R^{2}=0.59 \quad$ (t de $\quad$ Student entre parenthèses). Le délai moyen d'influence des variations de prix sur les variations de salaire se chiffre à $\bar{T}=\frac{0.69}{1-0.69}=2.22$ trimestres.
} 
dans toutes les branches des activités industrielles et dans toutes les régions, ce qui a incité Rueff à rechercher une cause générale, «une théorie précise susceptible d'être soumise à l'épreuve des faits ». A cette fin, Rueff (1925) présente un graphique montrant une connexion saisissante entre l'évolution du taux de chômage et celle du salaire réel moyen sur la période 1919-25, graphique qu'il a étendu jusqu'en 1930 dans son article de 1931 : «Il suffit alors de considérer simultanément ces deux courbes pour constater, sans que le doute soit possible, l'entier synchronisme de leurs mouvements respectifs. L'indice du chômage varie en général en même temps que le rapport salaires/prix sauf en quelques points (1er trimestre 1920, 1er trimestre 1924) où les variations du premier font suite immédiatement à celles du second». La figure 2 montre cette liaison à partir des données fournies dans l'Annexe I de l'article de 1931. ${ }^{10} \mathrm{Il}$ est à souligner que le chômage est corrélé positivement avec le salaire réel mais négativement avec le salaire nominal et les prix de gros, la première corrélation étant en outre sensiblement plus élevée en valeur absolue que les deux suivantes, suggérant ainsi que le salaire réel est effectivement sur cette période la grandeur pertinente pour l'analyse du chômage. ${ }^{11}$ Il importe d'indiquer ici que Pigou (1957) a confirmé la robustesse de cette corrélation en ajoutant deux années au graphique de Rueff (1925), et surtout en montrant que la relation de la figure 2 subsiste avec autant de précision en remplaçant les prix de gros par l'indice du coût de la vie, ce dernier étant jugé plus représentatif. En fait, ce qui surprend Rueff, ce n'est pas tant l'existence d'une corrélation entre le chômage et le salaire réel que la qualité de cette relation : "Cette conclusion, quant au fond, n'est pas pour nous surprendre. Elle se dégageait déjà de nombreuses études qualitatives, antérieurement consacrées au problème qui nous occupe. Ce qui, en elle, toutefois, est extrêmement inattendu, c'est la précision même des vérifications que l'expérience fournit. Ce qui nous étonne donc, ce n'est pas que cette relation soit vraie, c'est que, dans le chaos des réactions individuelles dont la résultante constitue le phénomène économique, elle le soit à un tel point... Il y a là croyons nous, un enseignement précieux, autant sur la nature des lois économiques que sur les méthodes à l'aide desquelles on peut tenter de les mettre en lumière» (Rueff, 1925). ${ }^{12}$

Dès son article de 1925, Rueff s'interroge sur la signification de ce phénomène: «Il est très curieux, et en apparence contraire à toutes les lois économiques, que le niveau des salaires ait pu rester stable, alors que l'offre de travail dépassait aussi sensiblement la

\footnotetext{
${ }^{10}$ Le taux de chômage est ici égal au rapport entre le nombre des ouvriers inemployés appartenant à des syndicats et le nombre total des membres de ces syndicats (ne sont pas comptés parmi les chômeurs les ouvriers privés de travail par maladie et les grévistes).

${ }^{11}$ Les coefficients de corrélations entre le taux de chômage $(U)$, le salaire réel $(S / P G)$, le salaire nominal $(S)$ et le niveau des prix de gros $(P G)$ sont les suivants (nous avons constaté que la considération des logarithmes améliore légèrement les corrélations):

$\begin{array}{ccccc} & U & \log (S / P G) & \log (S) & \log (P G) \\ U & 1.0 & 0.86 & -0.29 & -0.71 \\ \log (S / P G) & . & 1.0 & -0.44 & -0.88 \\ \log (S) & . & . & 1.0 & 0.81 \\ \log (P G) & . & . & . & 1.0\end{array}$

${ }^{12}$ Rueff s'interroge sur la contradiction observée entre les mouvements des deux variables en 1926 et attribue ce constat à une grève survenue au cours de cette année : «...il ne pouvait en être autrement : pendant la grève, en effet, les variations de salaires n'exercent aucune action sur l'emploi de la main-d'œuvre. Bien plus même, quand la main d'ouvre continuant à travailler malgré les ordres de grève décroît, les employeurs sont disposés à payer plus cher les ouvriers qui n'ont pas abandonné leur travail. D'où l'opposition des deux courbes, opposition suivie en 1927 d'une période de réadaptation progressive » (Rueff, 1931, p.215).
} 
demande de main d'œuvre. L'anomalie, toutefois, n'est là qu'apparente $» .{ }^{13}$ L'auteur met en avant le comportement des syndicats d'ouvriers qui tendent naturellement à s'opposer à la baisse des salaires, mais surtout, il accuse la mise en place d'allocations versées aux chômeurs: «La discipline des trade-unions en premier lieu est, en Angleterre, exceptionnellement puissante et le régime du contrat de travail collectif plus généralisé que partout ailleurs. La tradition, toutefois, eût été insuffisante à maintenir la résistance des ouvriers sans travail aux inévitables mouvements de salaires si une politique de subsides aux chômeurs, aussi généreuse que coûteuse pour le pays, n'avait permis à ceux-ci de rester indéfiniment inoccupés plutôt que de transgresser les instructions syndicales » (Rueff, 1925). En effet, la puissance des syndicats anglais avait conduit le gouvernement britannique à instituer en 1911 le «National Insurance Act». Pilotée par Lloyd George (bien que libéral, ce dernier fut accusé par les conservateurs d'adopter des mesures socialistes influencées par les idées de W. Beveridge), cette loi mis en place un système d'assurance chômage et d'assurance maladie d'après lequel chaque salarié âgé de 16 à 70 ans doit cotiser. ${ }^{14}$ En retour, en cas de chômage (n'excédant pas 15 semaines par an) des indemnités sont versées (la dole) de même qu'en cas de maladie les frais médicaux seront couverts ; la gestion de ce système était confiée aux mutuelles, syndicats et compagnies d'assurance.

En fait, la dole n'a fonctionné pleinement qu'à partir de 1920 en distribuant aux chômeurs «des prestations du même ordre de grandeur que les salaires d'activité » (Rueff, 1931), et c'est pourquoi le système d'assurance chômage est considérée comme la cause fondamentale de la rigidité à la baisse des salaires. ${ }^{15}$ Ces comportements conduisent en effet à instituer une sorte de revenu minimal (nous dirions aujourd'hui un «salaire de réserve ») à partir duquel l'ouvrier préfèrera recevoir la dole plutôt que de travailler pour un salaire qui ne serait que faiblement supérieur à cette dernière ${ }^{16}$, ceci impliquant que les chômeurs touchant la dole protègent indirectement les travailleurs contre des baisses de salaires. Par conséquent, les salaires ne peuvent plus jouer le rôle de variable d'ajustement pour résorber l'écart entre l'offre et la demande de travail car les salariés peuvent bénéficier d'un «équivalent revenu » égal à cette indemnité augmentée de la satisfaction attachée au loisir, ce total constituant une valeur de référence sur laquelle viennent buter les négociations à la baisse des salaires. Cette analyse mène Rueff (1925) à conclure que «l'observation des faits permet d'affirmer que pendant toute la période ..., il a existé en Angleterre, entre le nombre des chômeurs et le rapport du niveau des salaires au niveau général des prix, une relation permanente, toute variation de la valeur de ce rapport entraînant sans délai une variation concomitante de l'indice $d u$ chômage ». Bien que cette phrase avance clairement l'idée d'une causalité allant

\footnotetext{
${ }^{13}$ D'après l'auteur, avant la mise en place de la dole, il existait un mécanisme d'ajustement entre l'offre et la demande de travail car les chômeurs étaient dans l'obligation «de trouver du travail pour subsister, et la concurrence qui s'établissait entre eux ... les conduisaient à consentir des baisses de salaires. La diminution ne pouvait pas ne pas se prolonger jusqu'à ce que toute la main d'œuvre disponible fût employée... » (Rueff, 1931, p.227-28). Les résultats des tests économétriques présentés ci-après (cf. tableaux 2 et 3 ) infirment l'hypothèse d'une influence significative du chômage sur le salaire réel, illustrant ainsi le fait que la dole empêche un ajustement à la baisse des salaires lorsque le chômage augmente.

${ }^{14}$ Rueff (1931) précise dans l'annexe I qu'environ les trois quarts des travailleurs cotisent obligatoirement.

${ }^{15}$ Selon Rueff, une telle rigidité n'existe pas pour le prix du capital, puisque le prix des obligations - qui détermine le taux d'intérêt - est fixé directement en fonction des offres et des demandes de titres sur un marché financier concurrentiel. Ajoutons qu'au cours des années 1920, le taux d'intérêt réel est resté assez stable en Angleterre autour d'un niveau moyen de l'ordre de 5\% par an (Pettinger (2012)) : le développement du chômage ne peut donc être expliqué par l'évolution du prix (coût) du capital.

${ }^{16}$ A l'inverse de Rueff, on peut noter que Robinson (1947) qui s'intéressait à l'imperfection des marchés, défendait l'idée que les allocations chômage sont un élément indispensable dans la mesure où elles permettent de donner du temps pour la recherche d'emploi qualifié.
} 
des salaires réels vers le chômage, l'auteur reste néanmoins plus nuancé dans son article de 1931 : «l'énoncé précédent n'implique pas l'existence d'un lien causal entre les variations de salaires ou des prix et celles du chômage. Nous constatons seulement que dans le passé, le chômage n'a jamais varié sans variations de même sens du rapport salaires/prix... ». En fait, Rueff voulait signifier que la cause « causante » du chômage est la rigidité institutionnelle à la baisse des salaires nominaux provoquée par la dole : «la cause véritable, c'est ici et toujours l'assurance-chômage qui a rendu l'adaptation impossible » (Rueff, 1931, p.240). L'auteur en veut pour preuve qu'en l'absence d'indemnités versées aux chômeurs "avant la guerre, et dans tous les pays, le processus se poursuivait jusqu'à complète disparition [de ce] chômage».

Rueff (1931) indique que, bien que très discutée à l'époque, sa thèse d'un chômage «permanent» a réussi à convaincre un petit groupe d'économistes éminents, tels que Stamp, Pigou, Beveridge, Loveday et Siegfried. En particulier, l'analyse économique de Rueff rejoint celle de Pigou d'après lequel seule la flexibilité des salaires est susceptible de faire disparaitre le chômage, ce qui a bien sûr pour corolaire le fait que les rigidités salariales sont une source de chômage. Par ailleurs, Rueff s'était interrogé sur la question de savoir si la corrélation positive mise en évidence en Angleterre entre le salaire réel et le taux de chômage (figure 2) est observée dans d'autres pays. A ce propos, l'auteur indique que M.J. Denuc a mené des travaux sur cette question et publié ses résultats dans le Bulletin de la Statistique Générale de la France (avril-juin 1930) tendant à confirmer cette corrélation pour d'autres pays, même si nous dit Rueff, des précautions s'imposent en raison du fait, qu'en dehors de l'Angleterre, la fiabilité des indices des salaires et des indicateurs de taux de chômage est discutable. Enfin, mentionnons que, un demi-siècle plus tard, dans un article publié dans Le Monde, Rueff (1976) confirmait l'existence d'une corrélation positive entre le salaire réel et le taux de chômage pour la France sur la période 1963-1975 ; rappelons ici que, telle la dole, la mise en place du régime de l'assurance chômage en 1958 (Unedic et Assedic) a pu contribuer à générer du chômage «permanent». L'auteur concluait que, par rapport à l'Angleterre des années 1920, «la similitude prend toute sa valeur du fait qu'elle s'applique à une période de prix croissants. Elle montre la généralité du phénomène». Là encore, Rueff supposait implicitement la constance de la productivité, ce qui est d'autant plus dommageable que les statistiques officielles montrent des gains de productivité importants au cours de cette période. Nous verrons dans la partie 2 qu'Allais (1980) comblera cette lacune tout en soulignant le rôle des cotisations sociales dans la détermination du coût salarial dans la France des années 70.

\section{2 - Les débats des années 1920-30 autour de la «loi de Rueff», suivis de tests économétriques simples}

\section{Chômage «permanent » versus chômage « conjoncturel » : l'analyse de Pigou}

L'article de Pigou (1927) nuance l'interprétation de Rueff suivant laquelle le chômage anglais des années 1920 peut se résumer au seul chômage permanent. Même si Pigou confirmait la robustesse de la corrélation positive entre salaire réel et chômage en remplaçant les prix de gros par l'indice du coût de la vie et admettait l'idée d'un chômage «permanent », il suggère qu'un autre mécanisme a pu aussi contribuer significativement à l'explication. 
D'après l'auteur, la politique monétaire déflationniste menée par le gouvernement britannique pour défendre la livre - dans un contexte de désordre du système monétaire international, suite à l'abandon de l'étalon-or au début de la première Guerre et des tentatives de restauration de ce système pendant les années $1920^{17}$ - serait la cause majeure de la forte diminution du niveau des prix (cf. figure 1), ce qui renvoie bien sûr à la théorie quantitative ; en conséquence, compte-tenu du butoir constitué par la dole, cette baisse des prix génère une hausse mécanique du salaire réel. Jusqu'ici cette analyse ne contredit pas celle de Rueff. Cependant, le développement du chômage serait pour Pigou pour partie attribuable directement à la baisse des prix, phénomène défavorable aux marges bénéficiaires et donc aux activités de production. ${ }^{18}$ Autrement dit, la politique monétaire déflationniste serait un facteur commun ayant contribué à expliquer à la fois l'augmentation des salaires réels (par la baisse des prix) et celle du chômage. En bref, Pigou suggère qu'un chômage « conjoncturel » a pu s'ajouter au chômage «permanent » pour expliquer la «loi de Rueff», alors que Rueff conjecturait que ce type de chômage pouvait être négligé dans l'Angleterre des années 1920 (voir ci-après, §1.3).

\section{Salaire réel versus salaire nominal et l'hypothèse d'une causalité inverse allant du chômage vers le salaire réel : Rueff, Keynes et les observation de Dunlop \& Tarshi}

Un autre débat important porta sur la question «salaire nominal versus salaire réel » en liaison avec le niveau de l'emploi. Pour Keynes (1936, p.10), conformément à ce qui est observé pendant les années 1920 (voir ci-dessus, note (10)), le salaire nominal et le salaire réel évoluent en sens opposés : "This is because, in the short period, falling money wages and rising real wages are each, for independent reasons, likely to accompany decreasing employment; labour being readier to accept wage-cuts when employment is falling off, yet real wages inevitably rising in the same circumstances on account of the increasing marginal return to a given capital equipment when output is diminished". Autrement dit, pour un capital et des techniques donnés, la diminution de la production et donc de l'emploi impliquent une baisse des salaires nominaux, tandis que la hausse induite de la productivité du travail conduit à une hausse du salaire réel: ici, c'est l'augmentation du chômage qui conduit à la hausse des salaires réels. Cependant, contrairement à cette conjecture de Keynes, Dunlop (1938) et Tarshis (1939) ont montré, en considérant une longue période, que, le plus souvent, les salaires réels sont en Angleterre procycliques et qu'ils varient dans le même sens que les salaires nominaux. A ce propos, après avoir rappelé les résultats de Rueff $(1925,1931)$ et les commentaires de Pigou (1927), Keynes (1939, p.42) réagit en mettant en cause la signification statistique des résultats de Dunlop et Tarshis: "In the introduction to his 'Wages and Income in the United Kingdom since 1860', Prof. Bowley indicates that this is probably less than the margin of error for statistics of this kind. This general conclusion is reinforced by the fact that it is hourly wages which are relevant in the present context, for which accurate statistics are not available. Moreover, in the post-scriptum to his note, Mr. Tarshis explains that whilst real wages tend to move in the same direction as money wages, they move in the opposite direction, though only slightly, to the level of output as measured by man-

\footnotetext{
${ }^{17}$ Contrairement à Keynes, Rueff défendait l'étalon-or ; notamment, en assurant la stabilité des prix, ce systèmel permettait d'éviter une évolution incontrôlée des salaires réels.

${ }^{18}$ Le test de causalité prix-chômage présenté ci-après s'accorde avec ce point de vue (cf. tableau 2). En outre, l'année 1930 (début de la crise majeure des années 30), qui voit une forte baisse des prix de gros (et donc une hausse du salaire réel) et une baisse de la production accompagnées par une forte hausse du chômage, va dans le sens des idées de Pigou. Notons qu'avant les travaux du NBER d'après la seconde Guerre, l'Institut Harvard considérait les prix de gros comme un bon indicateur de la conjoncture économique.
} 
hours of employment; from which it appears that Mr. Tarshis's final result is in conformity with my original assumption, which is, of course, concerned with hourly wages. It seems possible, therefore, taking account of Mr. Meade's results ${ }^{19}$, that I may not, after all, have been seriously wrong ». Nous n'épuiserons bien sûr pas ici ce débat au sujet duquel Dos Santos Ferreira (1999) suggère que les résultats de Dunlop et Tarshis pourraient finalement aller dans le sens de l'argument de la Théorie Générale...

\section{Le remède au chômage : Rueff et Keynes}

Dans la «Théorie Générale », Keynes (1936) soulignait les conséquences d'une rigidité à la baisse des salaires nominaux sur le niveau de l'emploi, ce qui en principe rejoint l'hypothèse du chômage "permanent» de Rueff : la causalité salaire réel - chômage peut donc jouer dans les deux sens. Cependant, alors que pour Rueff la solution pour résoudre le chômage réside dans la suppression de la dole, pour Keynes, même sans cette dernière, la fixation des salaires nominaux ne résulte pas des mécanismes de marché évoqués par Pigou et Rueff, mais de conventions salariales entre les syndicats et les employeurs, signifiant ainsi une rigidité salariale intrinsèque au marché du travail. ${ }^{20}$ En outre, pour Keynes, la diminution du salaire ne résoudra pas automatiquement le chômage car la réduction induite de la demande sur le marché des biens conduit à une production insuffisante pour assurer le plein emploi. D'où l'insistance de Keynes sur la nécessité de garder des salaires élevés en période de dépression ; en outre, les travailleurs étant à court terme victimes de l'illusion monétaire, ils supporteront mieux une baisse des salaires réels si cette dernière résulte d'une hausse des prix que si elle résulte d'une baisse des salaires monétaires. En bref, par nature, le marché du travail keynésien ne possède pas le mécanisme autorégulateur infaillible décrit par Pigou et Rueff $^{21}$ et c'est pourquoi l'économie keynésienne est caractérisée par l'existence d'un équilibre de sous-emploi, situation où la mise en place par l'Etat d'une politique économique appropriée devient pleinement justifiée. Rueff (1947) avait pris vertement position contre les idées présentées dans La théorie Générale et, concernant le remède destiné à supprimer le chômage attribuable à des salaires réels excessifs, l'auteur s'oppose encore aux recommandations de Keynes dans son article de 1976 intitulé «la fin de l'ère keynésienne »: «Ainsi apparaît le grand secret de la pharmacopée keynésienne. Lorsque le niveau général des salaires est générateur de chômage, il faut, par majoration des dépenses d'investissement, provoquer une hausse du niveau général des prix». En fait, selon Rueff, l'accélération de

\footnotetext{
${ }^{19}$ Keynes se réfère ici à un rapport pour la Société des Nations rédigé par James Meade «World Economic Survey 1937-38», 1938, pp. 54-55 où ce dernier fait état d'observations allant dans le sens d'une corrélation négative entre salaires monétaires et réels (Meade fut le lauréat du prix Nobel d'économie en 1977).

${ }^{20}$ Pigou et Keynes s'étaient affrontés sur la question de la flexibilité du salaire monétaire (voir sur ce point la synthèse de Béraud (2003)). Pigou (1927, p. 357) relevait qu'avant la première guerre, la corrélation entre les salaires réels et le chômage était le plus souvent négative, suggérant ainsi que les mécanismes concurrentiels fonctionnaient sur le marché du travail en l'absence de dole: lorsque le chômage diminue (augmente), la position des salariés s'améliore (se détériore) pour demander des hausses de salaire, tandis que les employeurs sont plus (moins) incités à accorder ces hausses pour attirer la main d'œuvre nécessaire à la production.

${ }^{21}$ Notons cependant qu'une baisse simultanée des prix et des salaires conduirait - pour une offre de monnaie donnée - à une augmentation de la masse monétaire réelle. Si la consommation intègre un effet de richesse («effet Pigou »d'encaisse réelle), il peut exister un effet régulateur. Rueff était en sympathie avec cet « effet Pigou », mais nous ne sommes bien sûr plus ici dans le paradigme keynésien que Pigou combattait.
} 
l'inflation infligerait «de cruels sacrifices aux travailleurs, dont les revenus ne suivraient qu'avec retard la hausse des prix $»^{22}$

\section{Quelques éclairages économétriques sur le sens des causalités}

Les débats qui viennent d'être résumés illustrent bien le fait que la mise en évidence d'une corrélation positive entre les salaires réels et le chômage ne prouve pas que les premiers sont la cause du second; notamment, la conjecture d'une interdépendance conjoncturelle ayant pour facteur commun les prix (Pigou) ou celle d'une influence du chômage sur le salaire réel (Keynes) sont des hypothèses ne pouvant être écartées a priori, ces phénomènes n'étant d'ailleurs pas exclusifs les uns des autres. C'est pourquoi, afin d'apprécier dans quelle mesure on peut admettre avec Rueff que les salaires réels sont une cause du chômage, nous avons à nouveau procédé à des tests de causalité au sens de Granger (les variations des variables sont considérées afin de stationnariser les séries). Les résultats présentés sur le table $\mathbf{2}$ confirment avec force l'acceptation de l'hypothèse d'une influence positive du salaire réel sur le chômage alors que l'hypothèse inverse d'une influence du taux de chômage sur le salaire réel est rejetée sans ambiguïté (A). Le même test a été effectué entre les salaires nominaux et le chômage, puis entre les prix de gros et le chômage : les résultats conduisent à la conclusion (B) d'une absence de causalité allant des salaires nominaux vers le chômage mais à une possible causalité (négative) du chômage vers les salaires nominaux ${ }^{23}$ et à la conclusion (C) d'une causalité (négative) allant des prix vers le chômage, mais sans réciprocité. Dans l'ensemble, ces tests s'accordent donc avec l'hypothèse de Rueff suivant laquelle le chômage dépend des salaires réels et non l'inverse, tout en montrant que l'hypothèse d'une composante conjoncturelle liée à la baisse des prix n'est pas à exclure, comme le suggérait Pigou (1927). ${ }^{24}$

Table 2 - Analyse de la causalité au sens de Granger entre, d'une part, les salaires réels $(S / P G)$, les salaires nominaux $(S)$ ou les prix de gros $(P G)$, et d'autre part le taux de chômage $(U)$ - Angleterre : 1919.Q1-1930.Q4

(A) Les salaires réels causent (positivement) le chômage mais le chômage ne cause pas les salaires réels :

\begin{tabular}{ccrr} 
Null Hypothesis (2 lags) : & N. Obs & F-Satistic & Prob \\
\hline \hline$d(U)$ does not Granger Cause d $(\log (S / P G))$ & 45 & 2.16582 & 0.1279 \\
$d(\log (S / P G))$ does not Granger Cause d $(U)$ & & 20.3976 & $8 . E .07$ \\
\hline \hline
\end{tabular}

(B) Les salaires ne causent pas le chômage mais le chômage cause (négativement) les salaires :

\begin{tabular}{cccc}
\hline \hline Null Hypothesis (2 lags) : & N. Obs & F-Statistic & Prob. \\
\hline \hline $\mathrm{d}(\log (\mathrm{s}))$ does not Granger Cause d(U) & 45 & 2.00326 & 0.1482 \\
$\mathrm{~d}(\mathrm{U})$ does not Granger Cause d $(\log (\mathrm{S}))$ & & 4.67045 & 0.0150 \\
\hline \hline
\end{tabular}

\footnotetext{
${ }^{22}$ Voir encore Rueff (1951) qui, malgré la quasi-absence de chômage en France depuis la fin de la Seconde Guerre jusqu'aux années 1960, incitait les responsables de la politique économique à prendre en compte la relation entre le salaire réel et le chômage dans l'élaboration des règlementations du marché du travail. Cela n'a pas empêché la mise en place du système d'assurance chômage en 1958 et la hausse des cotisations sociales.

${ }^{23}$ Cette causalité reste plus incertaine que les autres, puisqu'elle disparaît au seuil de significativité de $1 \%$.

${ }^{24}$ En réalité, comme nous allons le voir, ce dernier type de chômage est aussi envisagé par Rueff (cf. §1.32).
} 
(C) Les prix causent (négativement) le chômage mais le chômage ne cause pas les prix :

\begin{tabular}{cccc}
\hline \hline Null Hypothesis (2 lags) : & Nb. Obs & F- statistic & Prob. \\
\hline \hline$d(\log (P G))$ does not Granger Cause $d(U)$ & 45 & 13.2449 & $4 . E-05$ \\
$d(U)$ does not Granger Cause d $(\log (P G))$ & & 2.48091 & 0.0965 \\
\hline \hline
\end{tabular}

Note table 2 - Les résultats présentés sont établis avec des retards allant jusqu'à deux trimestres. Cependant, on parvient à des mêmes conclusions avec des retards allant de 1 à 8 trimestres. Le signe indiqué de la causalité est celui des coefficients de régression.

Toutefois, si ces tests de causalité confirment que l'impulsion vient des salaires réels, l'explication du chômage avancée par Rueff fait intervenir les niveaux des variables et non leurs variations. C'est pourquoi nous avons complété l'exploration économétrique en régressant le taux de chômage sur sa valeur retardée et sur le salaire réel, et vice-versa : les résultats montrent que «tout se passe comme si » le taux de chômage dépendait des valeurs passées du salaire réel (table 3 (A)) avec un délai moyen d'environ un trimestre $\left(\bar{T}=\frac{0.53}{1-0.53}=1.13\right)$, alors que l'hypothèse inverse est à nouveau infirmée (table $\left.3(\mathbf{B})\right){ }^{25}$ Ajoutons ici qu'en remplaçant le salaire réel par le salaire nominal ou par le niveau des prix de gros dans la régression (A), ces variables n'apparaissent pas significatives pour expliquer le chômage. On peut donc conclure que, dans l'Angleterre des années 1920, «tout se passe comme si » les salaires réels expliquaient le chômage, et non l'inverse.

\section{Table 3 - Taux de chômage $(U)$ fonction des valeurs passées du salaire réel $(S / P G)$ versus $S / P G$ fonction des valeurs passées de $U$}

$$
\text { Angleterre - 1919.Q2 1930.Q4 }
$$

(A) $U$ dépend positivement des valeurs passées de $S / P G$ :

Dependent Variable: $U$

Method: Least Squares

Included observations: 47 after adjustments

\begin{tabular}{lrrrr}
\hline \hline \multicolumn{1}{c}{ Variable } & Coefficient & Std. Error & t-Statistic & Prob. \\
\hline \hline$U(-1)$ & 0.53 & 0.096 & 5.51 & 0.0000 \\
$\log (S / P G)$ & 12.2 & 2.58 & 4.73 & 0.0000 \\
$C$ & 3.4 & 0.79 & 4.24 & 0.0001 \\
\hline \hline R2 & 0.833 & Mean dependent var & 10.62 \\
Adjusted R2 & 0.826 & S.D. dependent var & 4.61 \\
S.E. of regression & 1.92 & Akaike info criterion & 4.21 \\
& & p-value LM test (2 lags) & 0.103
\end{tabular}

\footnotetext{
${ }^{25}$ Notons que l'infirmation de l'hypothèse d'une causalité allant du chômage vers le salaire réel illustre bien le fait que la dole empêche la baisse des salaires lorsque le chômage augmente.
} 
(B) $S / P G$ ne dépend pas des valeurs passées de $U$ :

Dependent Variable: $L O G(S / P G)$

Method: Least Squares

Included observations: 47 after adjustments

\begin{tabular}{lrcrc}
\hline \hline \multicolumn{1}{c}{ Variable } & Coefficient & Std. Error & t-Statistic & Prob. \\
\hline \hline $\log (S(-1) / P G(-1))$ & 0.859 & 0.085 & 10.13 & 0.0000 \\
$U$ & 0.004 & 0.003 & 1.29 & 0.2022 \\
\multicolumn{1}{c}{ C } & -0.010 & 0.025 & -0.404 & 0.6881 \\
\hline \hline R2 & 0.915 & Mean dependent var & 0.148 \\
Adjusted R2 & 0.911 & S.D. dependent var & 0.169 \\
S.E. of regression & 0.050 & Akaike info criterion & -3.07 \\
& & p-value LM test (2 lags) & 0.000
\end{tabular}

Note table 3 - Les estimations supposent que (A) le taux de chômage résulte des valeurs passées du salaire réel, les coefficients de pondération de ces valeurs décroissant exponentiellement avec l'éloignement du passé, (B) le salaire réel résulte des valeurs passées du taux de chômage, les coefficients de pondération de ces dernières décroissant exponentiellement (transformations de Koyck). Les $p$ values sont associées aux statistiques $F$ du test d'autocorrélation des résidus (Breusch-Godfrey serial correlation LM test) ; au seuil de 5\%, on peut conclure à l'absence d'autocorrélation pour l'équation (A) et à la présence d'autocorrélation pour l'équation (B).

\section{3 - Rueff a une vue plus large des facteurs du chômage que la seule «loi de Rueff »}

\subsection{1 - Le «rendement du travail » intervient dans le chômage «permanent »}

Concernant la corrélation positive entre le salaire réel et le chômage, Rueff écrivait dès son article de 1925 qu'il restait à en proposer une véritable théorie : «Nous avons négligé d'en présenter ici la théorie, impossible, croyons-nous, si on ne l'incorpore pas à l'étude générale de l'équilibre économique ». Dans son article de 1931, Rueff précise ses idées: «Il est indispensable de marquer que si des progrès techniques augmentaient d'une façon appréciable le rendement de la main-d'œuvre, le niveau du chômage s'en trouverait, toutes conditions égales, diminué. Mais en dépit des efforts de rationalisation, il est peu vraisemblable que les progrès techniques atteignent un niveau tel qu'ils permettent de réduire grandement le chômage»(p.221). Ces lignes montrent d'une part que Rueff suggère de comparer le salaire réel observé à un niveau d'équilibre ${ }^{26}$, d'autre part que ce niveau dépend de l'état des techniques, et enfin que sur la période analysée, l'évolution du progrès technique peut être négligée, ce qui revient à supposer constante la productivité du travail (Rueff évoque le « rendement actuel du travail », 1931, p.238). Sans même aborder la question complexe du caractère procyclique ou non de la productivité ${ }^{27}$, l'hypothèse de constance peut sembler a priori forte. C'est pourquoi nous avons calculé la productivité horaire du travail par le rapport entre les séries annuelles de la production nationale en volume et le nombre d'heures travaillées, ces statistiques étant données par Broadberry (1997) sur la période 1923-1930 ${ }^{28}$;

\footnotetext{
${ }^{26}$ Rueff intégrait systématiquement la notion d'équilibre dans ses analyses : «Ce double enseignement de Clément Colson et de Léon Walras m'avait apporté une autre notion capitale : celle de l'équilibre économique et des réactions de régulation propres à le maintenir »(Rueff (1977), p.36).

${ }^{27}$ Basu et Fernald (2001) analysent les raisons pouvant expliquer le caractère procyclique généralement observé de la productivité ainsi que les raisons incitant à la prudence dans cette conclusion.

${ }^{28}$ La Table A3 p.44 de l'ouvrage donne les valeurs de ces deux variables en base 1929=100.
} 
nous avons ensuite rétropolé ce rapport jusqu'en 1919 suivant le taux de croissance moyen annuel de la productivité estimé à $2.55 \%$ pour la période 1913-23 par Woltjer et de Jong (2010, Table 3, p. 14). La figure 3 représente sur la période 1919-1930 les évolutions du salaire réel moyen calculé par Rueff (1931) et de la productivité du travail ainsi approximée (données annuelles): cette dernière croit, mais moins rapidement que le salaire réel. La figure 4 compare sur la même période les évolutions annuelles du taux de chômage avec celle du logarithme du rapport salaire réel/productivité : non seulement la corrélation positive subsiste, mais encore elle est plus élevée (R2=0.91) que celle entre le taux de chômage et le logarithme $\mathrm{du}$ salaire réel $(\mathrm{R} 2=0.81)$. Ces résultats infirment donc l'hypothèse d'une productivité constante, mais ils confirment la thèse d'un chômage permanent attribuable à l'écart entre le salaire réel et son niveau d'équilibre.
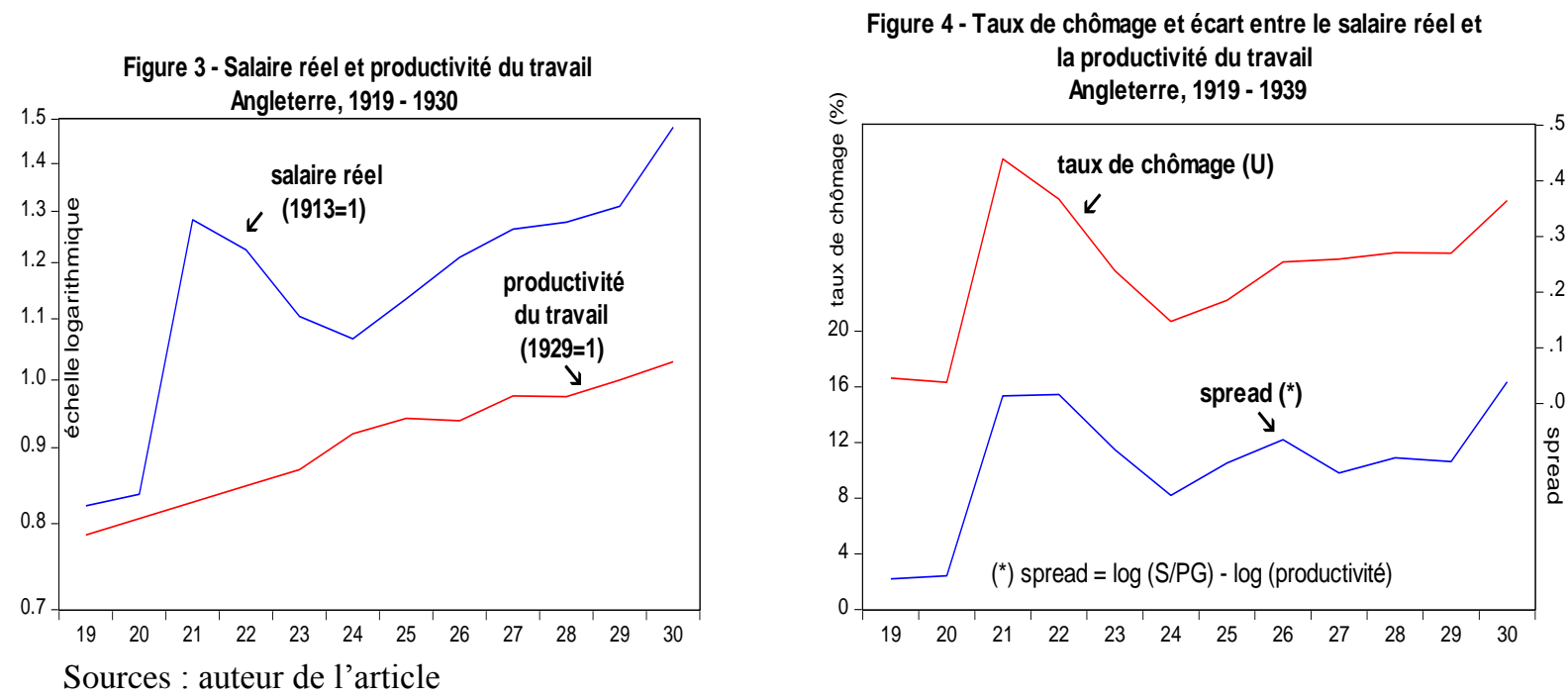

Le fait que la dole puisse créer une «trappe à inactivité » (expression non utilisée par Rueff) générant du chômage volontaire signifie bien sûr non pas la disparition de la fonction d'offre de travail mais une modification de cette dernière. En effet, le fait que la dole ne concerne pas de droit un quart des travailleurs et qu'elle ait une durée limitée, et le fait que les employés les plus qualifiés souhaitent recevoir un salaire significativement plus élevé que la dole, constituent autant de sources établissant un lien positif entre l'offre de travail et le salaire réel. Il y a donc bien chez Rueff l'idée classique d'un arbitrage entre le travail et le loisir permettant d'établir une fonction d'offre de travail croissante avec le salaire réel, et la vitesse de rotation élevée observée des chômeurs à cette époque montre la réalité de cette fonction. ${ }^{29}$ Autrement dit, le chômage «permanent » traduit à la fois du chômage volontaire et du chômage involontaire : le premier en raison de la «trappe à inactivité » liée au revenu de remplacement des chômeurs, le second en raison du fait que les chômeurs cherchant un emploi au salaire en vigueur n'en trouvent pas en raison d'un coût moyen du travail devenu excessif pour les entreprises eu égard au rendement de ce dernier. ${ }^{30}$ Il importe de relever ici que, si, dans la Théorie Générale, Keynes admettait - avec Marshall, Pigou et Rueff - le «premier postulat classique»d'après lequel le travail doit être rémunéré à sa productivité

\footnotetext{
${ }^{29}$ La durée du chômage est traitée dans le $\S d$ de l'Annexe III de Rueff (1931) où l'auteur constate une vitesse de rotation annuelle élevée des chômeurs, suggérant qu'une proportion significative de chômeurs désire à tout instant travailler. Cependant, il existe des chômeurs préférant leur situation à celle de travailleur, et «c'est cette 'masse irréductible' qui obère le plus lourdement le fonds d'assurance contre le chômage » (p.262).

${ }^{30}$ A ce propos, notons que Rueff considère que le salaire réel mesuré par le rapport entre le coût du travail $S$ et le prix de vente de la production $P G$ est représentatif du «prix relatif du travail» pour l'entreprise.
} 
marginale, par contre, contrairement à ces trois auteurs, il réfutait le «second postulat classique » d'un arbitrage travail-loisir, ce qui a pour conséquence d'éliminer le chômage volontaire, l'offre de travail keynésienne n'ayant plus de lien direct avec le salaire réel.

\subsection{2 - Rueff distingue trois types de chômage}

L'analyse économique et les faits amènent Rueff (1931) à distinguer conceptuellement trois catégories de chômage: le chômage «permanent», le chômage «temporaire » et le chômage «minimum ». ${ }^{31}$ Comme indiqué ci-dessus, le chômage «permanent » correspond à un excès durable du salaire réel attribuable à une rigidité institutionnelle du salaire nominal accompagnée d'une baisse des prix, ce type de chômage étant voué à disparaitre lorsqu'il n'existe pas de barrière au processus d'ajustement des salaires aux variations du niveau des prix.

Concernant le chômage «temporaire », Rueff pense qu'il peut se constater pendant les périodes de baisse cyclique des prix. Le fondement du chômage «temporaire » tient au fait que le niveau de l'activité économique peut être affecté par la baisse des prix ; en effet, il est probable qu' "une appréciation monétaire de trop grande amplitude conduise [certaines entreprises] à la faillite. C'est à ce titre que la baisse des prix peut affecter provisoirement l'activité économique et c'est l'une des raisons pour lesquelles il paraît souhaitable de réduire au minimum les variations du niveau général des prix»(p.240). De par sa nature, ce type de chômage ne peut perdurer car il est lié au cycle économique. On peut supputer ici que l'article de Pigou (1927) - suggérant l'existence d'un chômage conjoncturel lié à la baisse des prix - a pu influencer les idées de Rueff entre ses deux articles de 1925 et 1931. Dans l'Angleterre des années 1920, la baisse des prix (voir figure 1) pourrait donc a priori, dans la logique même de Rueff, avoir généré un chômage «temporaire »s'ajoutant au chômage «permanent », même si l'auteur pense finalement que ce chômage peut être négligé. A ce propos, les résultats du test de causalité prix-chômage présentés sur le Table 2 (C) ne contredisant pas l'hypothèse d'un chômage «temporaire » attribuable à la baisse des prix. Cependant, si l'on excepte les années 1920-21 pendant lesquelles la production a diminué, non seulement la baisse des prix n'a pas été accompagnée par une baisse de la production nationale, mais encore cette dernière s'est accrue à un rythme assez régulier dépassant $3 \%$ par an en moyenne, comme le montre la figure 5 comparant les évolutions des prix de gros et du revenu national réel sur la période $1919-1930^{32}$ : on peut donc raisonnablement en inférer que, même si du chômage «temporaire » a pu contribuer à la forte poussée du chômage en 192021 et à un moindre degré en 1930, la persistance du chômage massif et son développement durant la période 1919-30 ne peut être attribué à une baisse de l'activité économique induite par la déflation ou par tout autre facteur, ce qui naturellement renforce la thèse d'un chômage permanent en tant qu'explication principale. A ce propos, les résultats présentés dans l'Annexe I de cet article suggèrent, sur la base de l'équation d'Allais (cf. partie suivante), que si une composante conjoncturelle liée à la baisse de l'activité s'est ajoutée au chômage

\footnotetext{
${ }^{31}$ Rueff réfute d'autres causes souvent avancées à l'époque, dont notamment la thèse de «la fermeture des marchés », idée suivant laquelle les parts de marché des entreprises anglaises dans le monde auraient diminuées après la première guerre, notamment en raison du déclin de l'empire colonial britannique.

${ }^{32}$ Les valeurs du revenu réel sont tirées de l'ouvrage de Friedman et Schwartz (1982, Table 4.9), ces statistiques étant confirmées par les données fournies par Pettinger (2012) sur le «real GDP» britannique. La baisse d'activité constatée en 1926 s'explique par une grève (voir ci-dessus, note (13)). Une composante « temporaire » a sans doute aussi contribué à la hausse du chômage en 1930 (cf. note (19)).
} 
«permanent» au début des années 1920, cette composante a au contraire joué un rôle favorable à partir de 1924 en atténuant les effets massifs du chômage «permanent ».

Figure 5 - Prix de gros et revenu national réel Angleterre, 1919-1930

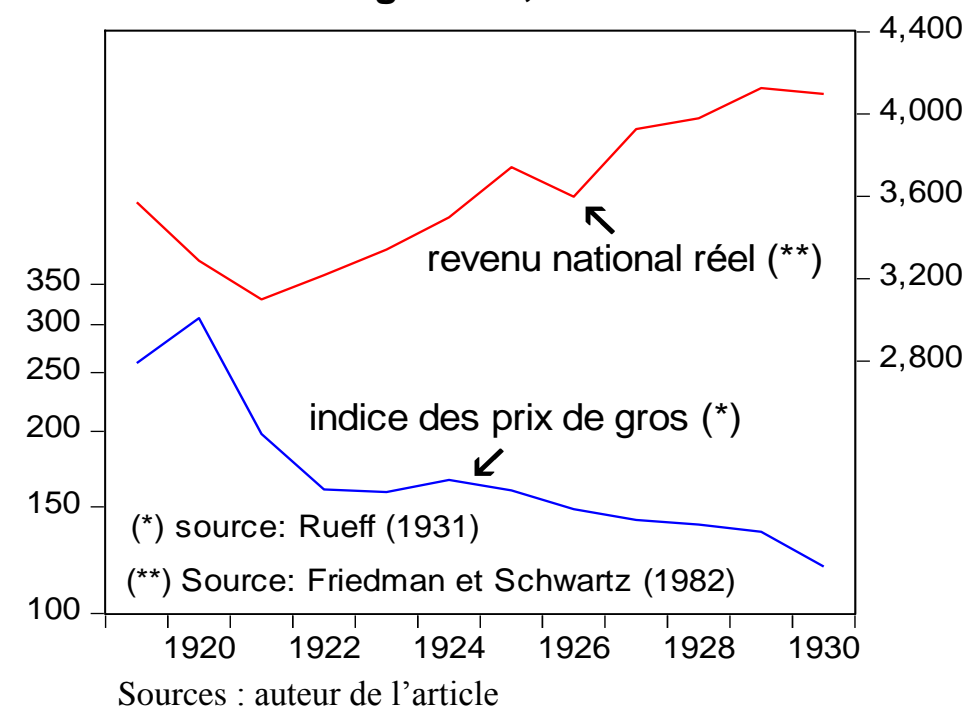

Dans la pensée de Rueff, l'idée que le chômage permanent doit finir par disparaître s'il n'y a pas de butoir institutionnel ne signifie pas pour autant la disparition du chômage, et ceci même en l'absence de chômage "temporaire ». En effet, Rueff avance aussi l'idée qu'il existe un "minimum de chômage semblant répondre, dans chaque pays, aux conditions normales d'emploi de la main d'œuvre » (Rueff, 1931, p. 228). L'auteur évoquerait sans doute aujourd'hui la notion de chômage «frictionnel ». En effet, dans l'annexe IV de son article de 1931, Rueff précise les phénomènes expliquant ce type de chômage ${ }^{33}$ : «mobilité $^{-}$ imparfaite de la main d'œuvre à la fois de région à région et d'industrie à industrie», «méthodes d'embauche défectueuses», «ignorance des conditions du marché du travail à la fois du côté de l'ouvrier en quête d'emploi et du côté de l'employeur», ce dernier phénomène ayant conduit à la création des Bourses de Travail en 1909 dont le but était de mettre en contact les ouvriers et les employeurs. On rejoint ici l'idée d'imperfection du marché du travail, attribuable notamment à une information imparfaite. ${ }^{34}$ Dans la mesure où il n'y a pas de raison particulière pouvant laisser penser que ce type de chômage ait sensiblement évolué dans l'Angleterre des années 1920, l'hypothèse implicite de Rueff est que ce chômage «minimum» ne saurait expliquer l'augmentation constatée du chômage.

\footnotetext{
${ }^{33}$ Les «variations saisonnières» sont également évoquées par Rueff parmi les causes «normales ».

34 Le chômage dit frictionnel renvoie aux modèles de «Job Search» (Stigler 1962) et aux modèles d'appariement ou «Matching » (Pissarides, 1990). On peut également évoquer la courbe de Beveridge traduisant une corrélation négative entre le nombre de chômeurs et celui des emplois vacants : en période de faible chômage, les offres d'emplois non satisfaites sont relativement nombreuses car la tension sur le marché du travail est accompagnée par une inadaptation des offres aux demandes d'emplois plus importante qu'en période de sous-emploi massif.
} 


\section{2 - Allais et les trois composantes du chômage distinguées par Rueff}

Polytechnicien tout comme Rueff, Allais fut - contrairement à son prédécesseur reconnu au plus haut niveau académique, puisque le prix de la banque de Suède en mémoire d'Alfred Nobel lui fut décerné en 1988 pour récompenser ses travaux théoriques sur les marchés et l'allocation optimale des ressources. Une des pièces maitresses de l'œuvre d'Allais est représentée par son Traité d'économie pure où l'auteur présente dans le chapitre V une analyse la relation entre salaires réels et chômage. ${ }^{35}$ Cette analyse conduit l'auteur à la conclusion que «dans une économie différenciée ${ }^{36}$ de concurrence et pour un équipement matériel, des techniques et une durée légale du travail données, une élévation permanente du taux des salaires réels aura pour effet durable une restriction permanente de l'emploi, c'està-dire un chômage permanent » (Allais, 1994 [1943], p. 695). Si dans son Traité Allais reprend à son compte l'appellation de «chômage permanent» (sans pour autant se référer à Rueff sur cette terminologie), il y employait aussi l'expression de «chômage chronique » (p.698) que l'on peut considérer comme synonyme. Dans la note 4, de la p.703 du Traité, Allais vise d'une manière lapidaire l'article de Rueff (1925) pour indiquer que l'exemple de l'Angleterre des années 1920 confirme l'existence d'un chômage «chronique $»{ }^{37}$ L'analyse d'Allais est générale, mais l'auteur souligne le rôle particulier que peuvent jouer les indemnités chômage, rejoignant ainsi directement l'analyse de Rueff : «... plutôt que de travailler, un certain nombre d'individus préfèreront chômer en se contentant du standard de vie réduit que leur assure leur indemnité de chômage et il est visible que leur nombre sera d'autant plus élevé que le montant de l'allocation sera plus grand par rapport au minimum vital. On voit ainsi que l'institution d'une allocation chômage aura pour effet de créer un nouvel équilibre comportant un chômage permanent. Nous conviendrons d'appeler 'chômage volontaire' le chômage de ceux qui préfèrent se contenter des allocations de chômage plutôt que de travailler au taux du marché» (p.700). Autrement dit, tout comme le chômage permanent de Rueff, le chômage chronique d'Allais est un chômage d'équilibre pouvant inclure à la fois du chômage volontaire et du chômage involontaire attribuable à des charges salariales excessives. Toutefois, alors que Rueff se réfère à des rigidités nominales génératrices d'un excès de salaire réel, l'approche d'Allais admet directement l'existence d'un «salaire global réel» rigide incluant les charges sociales. Deux autres catégories de chômage sont aussi considérées par Allais dans son Traité (note (14'), p.698) : le "chômage cyclique des dépressions économiques correspondant aux crises périodiques» et le «chômage d'adaptation dû̀ à l'inertie d'une économie inadaptée en évolution ». Au total, on voit donc que, dès 1943, Allais considérait les trois sources de chômage évoquées par Rueff. Dans le droit fil de son exposé théorique du Traité, Allais $(1980,1981)$ s'intéressa beaucoup plus tard à la forte augmentation du chômage français des années 1970. Dans l'introduction à la troisième édition du Traité, en 1994, l'auteur résume ainsi les idées sous-jacentes à sa contribution empirique de 1980 : «Le point de départ de mes recherches a été ma théorie du

\footnotetext{
${ }^{35}$ Voir section B de ce chapitre. Dans la note (1), p.694, Allais indique que le point de départ de ses développements consacrés à la relation salaire réel - chômage a été l'ouvrage de James Meade (1939, pp.106 et suiv.).

${ }^{36}$ Allais généralise ici, pour une économie où plusieurs entreprises existent par secteur d'activité, le résultat obtenu dans une économie il n'existe qu'une seule entreprise par secteur.

${ }^{37}$ On doit aussi relever que dans son polycopié associé à son enseignement donné à l'Université de Paris Ouest, Allais (1971, p.508) faisait état du graphique publié par Rueff en 1925 montrant les évolutions parallèles du salaire réel et du taux de chômage en Angleterre entre 1919 et 1925 (figure 2 ci-dessus), et indiquait en bibliographie l'article publié par Rueff en 1931, mais sans aucun commentaire.
} 
chômage telle que je l'ai exposé dans mes différents travaux, à la suite de mon ouvrage de 1943. Suivant cette analyse, le chômage de la période 1952-1978 [en France] résultait pour l'essentiel de trois causes majeures : des charges salariales trop élevées relativement à leur valeur d'équilibre (chômage chronique), des fluctuations conjoncturelles (chômage conjoncturel), et du progrès technique (chômage technologique). Mon analyse de 1980 a été effectuée dans le cadre d'un modèle économétrique à partir d'hypothèses relativement simples et elle a donné d'excellents résultats ». (Allais, 1994, [1943], p. 72).

Concernant ces trois catégories de chômage, il est d'autant plus étonnant qu'Allais ne se soit pas référé explicitement à Rueff que ce dernier venait de publier en 1976 un article dans Le Monde confirmant la «loi de Rueff» pour la France sur la période 1963-1975. ${ }^{38}$ Rueff mourut en avril 1978, soit deux ans à peine après son article de 1976, et n'avait donc malheureusement pas pu connaître l'article publié par Allais en 1981 dans ce même quotidien où ce dernier faisait état des résultats de l'estimation de son équation économétrique simple du chômage en France basée sur les trois catégories de chômage distingués par Rueff. ${ }^{39}$ En fait, les résultats présentés par Allais étaient le fruit d'un modèle préalablement détaillé dans un rapport d'activité scientifique (non publié) présenté en 1980 au CNRS (i.e. le «modèle $M C$ » pour « Modèle du Chômage ») où l'auteur affirmait que «Le chômage de plus en plus important qui s'est constaté en France depuis 1968 a donné lieu à une immense littérature, mais de cette littérature, aucune étude valable n'a émergé qui précise d'une manière quantitative les facteurs déterminants essentiels du chômage sur l'ensemble de la période d'après guerre» (p.46). On ne peut ici que regretter le fait que cette appréciation générale n'ait pas été accompagnée d'une critique précise des contributions de la littérature.

Allais présente le chômage «chronique » dû à une rigidité à la baisse des salaires avec la figure 6 qui est une variante d'un schéma très classique mais qui a le mérite d'illustrer pourquoi le chômage peut résulter d'un «salaire global réel » trop élevé par rapport à sa valeur de l'équilibre concurrentiel (cotisations sociales comprises) : alors que la différence $\xi_{e}-\xi$ correspond au chômage «effectif» engendré par l'excès du salaire réel sur sa valeur d'équilibre (segment $R Q$ ), le segment $R S$ traduit du chômage «apparent » correspondant à l'excès observé des demandes sur les offres d'emplois. Ce chômage «apparent » correspond au chômage chronique «...qui se constaterait en l'absence de toute fluctuations conjoncturelle et de tout progrès technique. C'est un chômage d'équilibre, qui résulte de rigidités structurelles .... Il s'ensuit que la composante représentative du chômage chronique ne présente aucune fluctuation conjoncturelle »(Allais, 1980, p.48). Lors des estimations

\footnotetext{
${ }^{38}$ Il ne faut sans doute pas voir là une volonté délibérée de l'auteur d'occulter les apports de Rueff, car, comme plupart des économistes, tout de passe comme si Allais ne connaissait que le chômage «permanent» de Rueff illustré par la «loi de Rueff» et ignorait donc que ce dernier admettait aussi la possibilité d'un chômage «transitoire » et d'un chômage « minimal».

${ }^{39}$ Gomez (2012) a suggéré un lien entre Rueff et Allais en ce qui concerne le chômage attribuable à des salaires réels trop élevés. En outre, en se référant aux analyses de Patrick Artus portant sur différents pays (cf. les «Flash Economie " d'Artus accessibles sur internet), Gomez montre que les Etats-Unis, l'Allemagne et surtout le Royaume-Uni apparaissent actuellement comme les pays où les rigidités de salaires sont les plus faibles (corrélations négatives marquées entre les variations du chômage et des salaires réels), alors que l'Italie, l'Espagne et la France apparaissent comme les pays à fortes rigidités (faibles corrélations négatives significatives ou corrélation positive), la France étant considérée par l'auteur comme «à la traine » en ce qui concerne les réformes qui pourraient rendre le marché du travail plus flexible. En fait, l'approche en termes de variations ne paraît pas conforme aux idées de Rueff et d'Allais pour lesquels le niveau du salaire réel doit être considéré. Pour Allais, le salaire réel varie à court terme dans le même sens que la production, de sorte qu'une augmentation peut temporairement être associée à une diminution du chômage.
} 
économétriques, Allais admet ainsi qu' «au voisinage d'une situation d'équilibre, le taux de chômage constaté (chômage apparent) est proportionnel [au trend de] ... l'excès du logarithme du salaire réel global sur sa valeur d'équilibre» (Allais, 1980, p.49**). On doit souligner ici que l'hypothèse implicite de constance du «rendement du travail » qui était admise par Rueff (correspondant à la valeur du salaire réel d'équilibre $S_{G e} / P$ sur la figure (6)) est relâchée par Allais car elle n'est a priori pas admissible à long terme dans une économie conditionnée par un rythme soutenu de progrès technique telle que le France.

Figure 6 - Illustration du chômage « chronique » suivant Allais

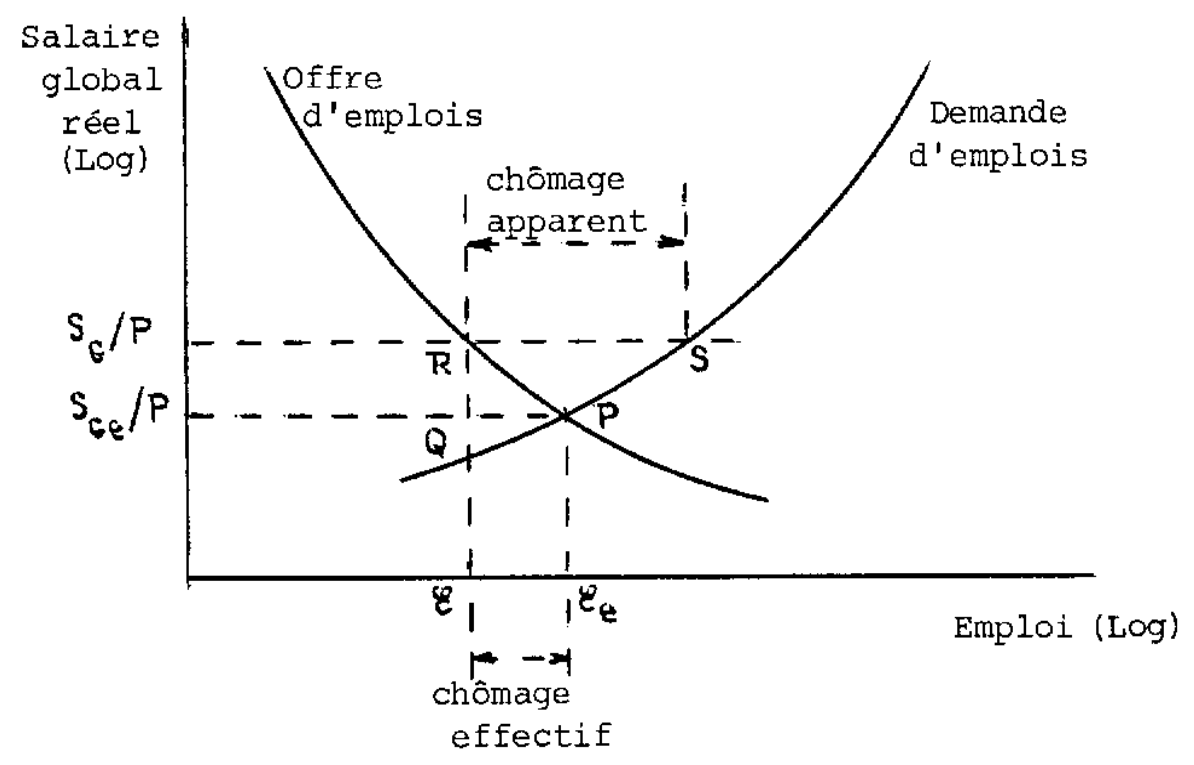

Source - Allais, 1980, p. 49**. $S_{G} / P:$ salaire global réel effectif (charges sociales obligatoires comprises); $S_{G e} / P \quad:$ valeur d'équilibre de $S_{G} / P$, égale à la productivité marginale du travail.

Concernant le chômage «conjoncturel», il est la conséquence des fluctuations de l'activité économique, que ces dernières aient des causes endogènes ou exogènes. Allais suppose ainsi que la partie de l'évolution du chômage non expliquée par la composante chronique est proportionnelle à l'écart au trend de la production. ${ }^{40}$ Le statut théorique de cette composante conjoncturelle n'est pas vraiment précisé par l'auteur, mais on peut penser que ce dernier y voyait plus un moyen pour représenter le chômage conjoncturel qu'un facteur explicatif à proprement parlé, ce qui n'est pas sans rappeler la «loi d'Okun » (1962)). Suivant cette optique, les facteurs explicatifs sont implicitement considérés comme sous-jacents aux fluctuations de la production. Un point important considéré par Allais - et non par Rueff - est la distinction entre les effets chroniques et les effets conjoncturels du salaire réel sur l'emploi : «...on constate généralement que le chômage conjoncturel diminue lorsque les salaires réels augmentent... le modèle $M C$ a précisément pour objet de définir une technique appropriée pour dissocier ces deux effets dont la nature est entièrement différente... faute de

\footnotetext{
${ }^{40}$ En fait, il peut sembler qu'il aurait été conceptuellement préférable de considérer la marge de production disponible qui est par construction toujours positive ou nulle ; cependant, à une constante de calage près, cette marge peut être approximée par l'écart au trend de la producion.
} 
les distinguer, l'analyse des causes effectives du chômage a donné lieu à des controverses aussi passionnées que stériles »(Allais, 1980, p. 48, note (3)). Nous rejoignons ici le débat résumé ci-dessus où Keynes s'opposait à Rueff en soutenant l'idée qu'une hausse des salaires pouvait avoir des effets favorables sur l'emploi en stimulant la demande : la réponse d'Allais se trouve donc dans la distinction entre des effets défavorables à long terme mais favorables à court terme, ces effets étant implicitement capturé par l'écart au trend de la production. En fait, pour Keynes, comme pour Allais, un écart persistant entre le salaire réel et la productivité est un état de la nature conduisant à un chômage durable ; cependant d'après Keynes, une hausse du salaire nominal peut stimuler la demande de biens et ainsi générer des hausses de prix pouvant in fine abaisser temporairement le salaire réel, favorisant ainsi l'emploi dans le court terme, ce qui semble compatible avec la vision d'Allais.

Enfin, Allais distingue une troisième source génératrice de chômage : « Le chômage technologique résulte des mutations qu'impose le progrès technique et des délais d'adaptation de l'économie à ces mutations; c'est le prix qu'il faut payer pour le progrès (Allais, 1980, p.48)... le chômage technologique peut être supposé comme sensiblement constant ... » (p.49) ». ${ }^{41} \mathrm{Au}$ total, les composantes, chronique $\left(U_{1 t}\right)$, conjoncturelle $\left(U_{2 t}\right)$ et technologique $\left(U_{2 t}\right)$ du chômage s'expriment très simplement (Rueff aurait évoqué ici les composantes permanente, temporaire et minimale) :

$$
\begin{aligned}
& U_{1 t}=\lambda\left[a \frac{y_{t}-y_{1}}{y_{1}}+b\right]^{42}, \quad U_{2 t}=\mu \log \frac{Q_{t}}{Q_{T t}}, \quad U_{3 t}=u_{0} \\
& \lambda>0, \quad a>0, \mu<0, u_{0}>0
\end{aligned}
$$

$y_{t}$ : trend de $\left(S_{t} / P_{t}\right) / \prod_{t}$ (moyenne mobile sur 5 ans), $y_{1}$ : valeur de $y_{t}$ en 1960 (traduit l'hypothèse d'absence de chômage chronique à cette date)

$S_{t}$ : indice du coût de la main d'œuvre dans les industries mécaniques et électriques (charges sociales comprises)

$P_{t}$ : indice du niveau général des prix (déflateur du PIB)

$\Pi_{t}$ : indice de productivité nette du travail

\footnotetext{
${ }^{41}$ Dans son ouvrage de 1999, Allais décompose le chômage en cinq catégories : chômage chronique, chômage dû au libre échange mondialiste, chômage dû à l'immigration, chômage dû au progrès des technologies et enfin chômage conjoncturel. Avouons que l'approche proposée dans cet ouvrage pour évaluer en France sur la période 1975-97 ces différentes catégories de chômage nous est apparue comme très arbitraire et n'a pas emporté notre conviction.

${ }^{42} \mathrm{Si}$ on comprend bien le rôle du paramètre $\lambda$ eu égard à la figure 6 (le chômage chronique est proportionnel à l'excès de salaire réel), Allais n'explique pas les raisons pour lesquelles il introduit les paramètres $a$ et $b$ (l'excès de salaire réel n'est pas directement mesuré par le rapport $\frac{y_{t}-y_{1}}{y_{1}}$ mais par une fonction linéaire de ce rapport). On peut penser que ces deux derniers paramètres peuvent capturer des éventuels biais de mesure associés aux indicateurs statistiques retenus et/ou moduler l'arbitraire dans le choix de l'année 1960 pour fixer la valeur de $y_{1}$; en fait, $b$ capture aussi un calage entre l'écart au trend (dont la moyenne est nulle) et la marge de production disponible (dont la moyenne est positive) qui aurait été a priori conceptuellement mieux appropriée. On remarque que, sous la condition $b=0$, la constante estimée $C_{0}$ représente la composante technologique du chômage. Par ailleurs, notons que le rapport $\frac{y_{t}-y_{1}}{y_{1}}$ est, à une constante près, peu différent de
} $\log \left(\left(S_{t} / P_{t}\right) / \Pi_{t}\right)$ représentée sur la figure 4 (Angleterre des années 1920). 
$Q_{t}$ : indice de la production industrielle

$Q_{T t}:$ trend log-linéaire de la production industrielle

En supposant que les trois composantes du chômage sont additives, l'auteur confronte l'équation économétrique simple suivante aux données de l'observation :

$$
\begin{aligned}
& U_{t}=\underset{>0}{A} \frac{y_{t}-y_{1}}{y_{1}}+\underset{<0}{B} \log \frac{Q_{t}}{Q_{T t}}+C_{0}+\varepsilon_{t} \\
& A=\lambda a, \quad B=\mu, \quad C_{0}=\lambda b+u_{o}
\end{aligned}
$$

où $U_{t}$ est le taux de chômage mesuré par le rapport de la "population disponible à la recherche d'un emploi » à la «population active », $\varepsilon_{t}$ étant un terme d'erreur. D'après Allais, le trend du rapport entre le salaire réel (salaire augmenté des cotisations salariales et patronales) et la productivité du travail explique la tendance du taux de chômage: "L'idée directrice ...est que la variable réellement importante pour expliquer le chômage chronique est le rapport des salaires réels globaux à la productivité nette »(Allais, 1980, p.50). Malgré sa simplicité, l'auteur montre avec la figure 7 que l'équation (1) représente assez bien l'évolution du taux de chômage en France sur la période 1952-1978, et en particulier le décrochage des années 1973-75 ( $c$ et $c^{*}$ sont les valeurs observées et calculées sur données annuelles du taux de chômage) ; les valeurs estimées des coefficients $A=7.25, B=-8.69$ de l'équation (1) sont significatifs et ont les signes attendus (la valeur de la constante $C_{0}=1.19$ est a priori quelconque en raison notamment de l'indétermination du signe du paramètre $b$ ); en outre, l'auteur montre qu'un découpage en deux sous-périodes conduit à des coefficients pouvant être supposés stables. L'auteur conclut en attribuant aux charges salariales totales (comprenant les cotisations sociales obligatoires) un rôle important en tant que facteur de rigidité du coût du travail : "au regard de l'analyse effectuée et des résultats obtenus, il est certain qu'une partie importante du chômage français est due à des charges salariales moyennes excessives relativement au niveau qui serait le leur s'il n'existait aucune rigidité structurelle » (Allais, 1980, p.57). ${ }^{43}$ Cependant, les propriétés statistiques des résidus de l'équation ne sont malheureusement pas analysées alors que, au regard des écarts observables sur la figure 7, il est visible que ces derniers ont une structure autorégressive, suggérant ainsi un défaut de spécification ou une incomplétude des facteurs.

\footnotetext{
${ }^{43}$ Allais (1980, p.53) indique que des résultats comparables ont été obtenus aux Etats-Unis et en Italie sur des périodes voisines par ses doctorants (Christian Gomez et Louis Sina respectivement).
} 


\section{Figure 7 - Valeurs observées et valeurs calculées d'après le « modèle MC » d'Allais}

France, 1952 -1978

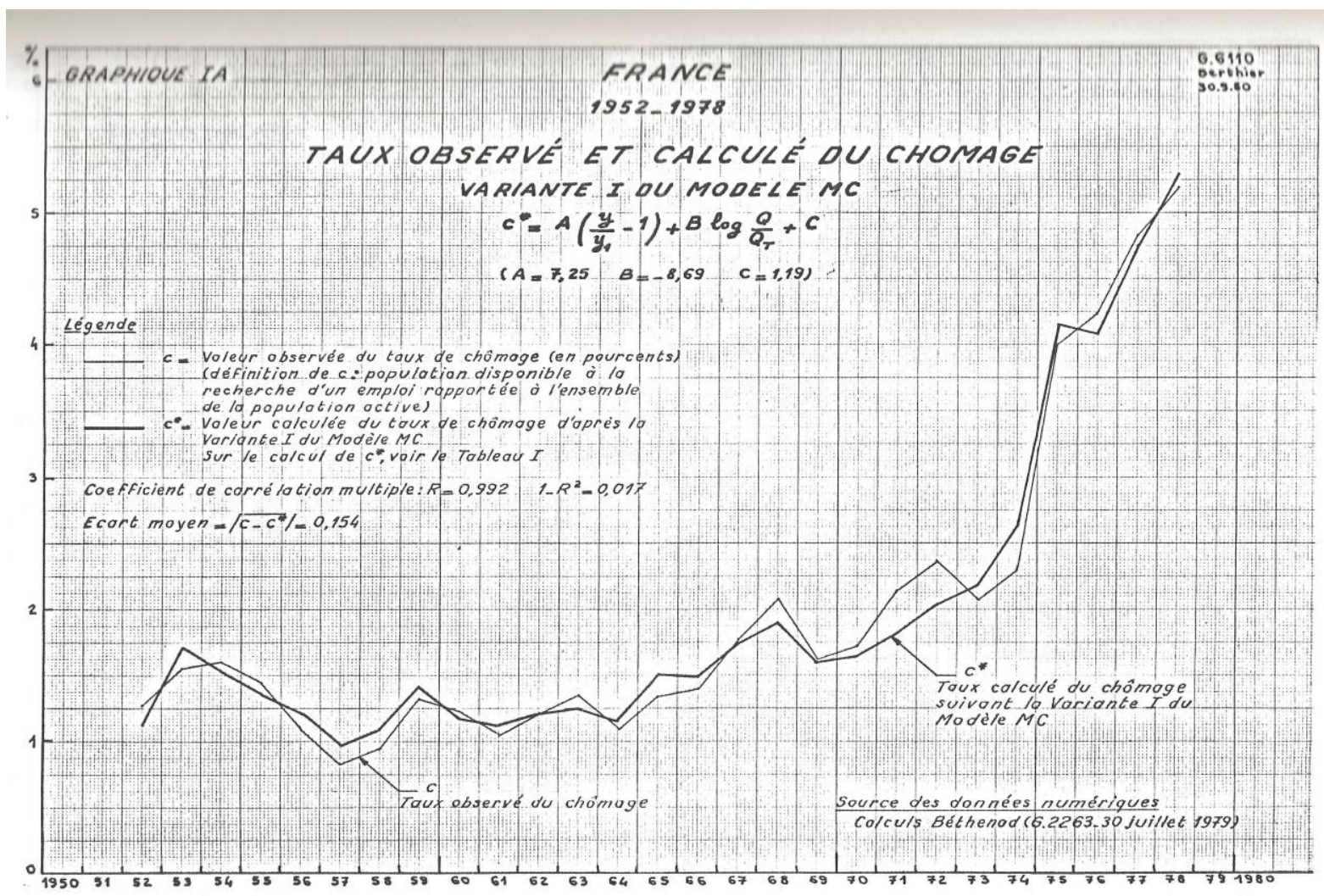

Source : Allais (1980), p. 61

On voit donc qu'Allais (1980) confirme l'approche de Rueff en tenant compte explicitement de la productivité du travail et des fluctuations de l'activité économique, et en supposant constante la part du chômage attribuable à des facteurs technologiques : ces trois phénomènes avaient été visés mais non pris en compte dans les vérifications empiriques présentées par Rueff (cf. ci-dessus, § 1.3). Nous avons estimé l'équation d'Allais en France sur la période 1970-2008 en utilisant une série plus représentative des charges salariales globales (i.e. ne se limitant pas aux industries mécaniques et électriques). Les résultats obtenus présentés dans l'Annexe 1 confirment que les principales tendances du chômage sont approximativement représentées; cependant, il existe des résidus importants ayant des propriétés indésirables (autocorrélation, hétéroscédasticité) confirmant un défaut de spécification ou une incomplétude de cette équation, ce qui n'est guère étonnant compte tenu de sa simplicité.

Pour finir, on doit relever que Rueff invoquait les indemnités versées aux chômeurs pour expliquer le chômage «permanent » dans l'Angleterre des années 1920, alors qu'Allais invoquait plus généralement les charges salariales globales dans le développement du chômage «chronique » français des années 1970, ces dernières comprenant les salaires et les cotisations sociales. Cependant, comme indiqué ci-dessus, le chômage volontaire attribuable à 
l'assurance chômage constitue pour Allais un élément implicitement contenu dans le chômage chronique pouvant être attribué aux indemnités versées aux chômeurs (le système d'assurance chômage a été institué en France en 1958) : en amputant l'offre «active» de travail, les allocations ne peuvent que favoriser le maintien (ou même la hausse) des salaires et ainsi contribuer à l'excès de salaire réel. Concernant les cotisations sociales obligatoires, elles étaient beaucoup plus faibles dans le cas anglais des années 1920 que dans l'exemple français des années 1970, ce qui explique que Rueff n'ait pas, à notre connaissance, évoqué d'une manière explicite ces charges obligatoires. ${ }^{44}$ Dans son article du Monde publié en 1976, l'auteur voyait dans l'exemple français des années 1963-1975 une confirmation des résultats obtenus dans l'Angleterre des années 1920, ce qui suppose implicitement que les allocations chômage évoluent à peu près proportionnellement aux salaires; notons ici que, de même qu'il aurait pu facilement relâcher l'hypothèse d'une productivité constante, Rueff aurait pu ajouter les cotisations sociales à son indicateur de salaire réel, et il faut constater qu'il n'a fait ni l'un ni l'autre.

\section{3 - Rueff et Allais revisités sous le prisme du modèle WS-PS}

Entre le milieu des années 1970 et la fin des années 1980, une littérature de nature microéconomique a montré que les rigidités salariales peuvent être le résultat de comportements conjoints des employeurs et des salariés; ces rigidités intervenant dans un cadre d'analyse ayant des propriétés classiques, cette littérature est qualifiée de «néokeynésienne $»^{45}$ Il en va ainsi de la théorie du salaire d'efficience (Shapiro et Stiglitz, 1984), du modèle Insider-Outsider (Lindbeck et Snower, 1989) et de la théorie des contrats implicites (Azariadis, 1975). En introduisant l'hypothèse d'une information asymétrique entre salariés et employeurs, ces modèles montrent que les comportements d'agents rationnels et adverses au risque peuvent conduire à des situations non-concurrentielles empêchant l'ajustement du salaire réel vers son niveau d'équilibre, suggérant ainsi la possibilité d'un chômage de type «permanent » ou «chronique ». ${ }^{46}$ Cependant, par rapport à la pensée de

\footnotetext{
${ }^{44}$ En fait, puisque pour représenter le salaire moyen Rueff utilisait un «indice général des prix du travail », cela suggère que cet indice intègre ces cotisations qui étaient en totalité payées par les salariés.

${ }^{45}$ Nous n'aborderons pas ici les nombreux développements de la littérature issus de la courbe de Phillips (1958), cette dernière traduisant initialement une corrélation négative entre le chômage et les variations du salaire nominal. Rueff (1976) avait vertement rejeté cette approche : "Je ne m'attarderai pas à discuter ici la soi-disant "relation de Phillips", qui étudie le marché du travail sans considération des prix auxquels le travail est vendu. Comme il était prévisible, elle s'est révélée fausse et sans portée ». Alors que Rueff n'avait pu connaître le concept de NAIRU proposé en 1980 par Tobin (non-accelerating inflation rate of unemployment), tout se passe comme si Allais s'en était désintéressé.

${ }^{46}$ On peut aussi rappeler la «théorie du déséquilibre » (Patinkin (1956), Clower (1965)) qui est ancrée dans la théorie walrassienne de l'équilibre général et qui s'est vite transformée en «théorie des équilibres temporaires à prix fixes » (Barro-Grossman 1971, Bénassy 1975, Drèze 1975). Suivant ce paradigme, il peut exister à la fois sur le marché du travail et celui des biens des situations de déséquilibre entre l'offre et la demande, les autres marchés étant supposés équilibrés. Les prix et les salaires étant supposés fixes à court terme, les ajustements s'effectuent par les quantités, ce qui assure la cohérence du système. Dans ce cadre, les contributions ont montré la possibilité, suivant les périodes, d'un chômage "classique » ou d'un chômage « keynésien » (cf. notamment Malinvaud (1977)). A la fois le chômage classique et le chômage keynésien peuvent être rapprochés du chômage «permanent» de Rueff du le chômage «chronique »d'Allais, puisque dans les deux cas un salaire réel plus élevé que son niveau concurrentiel génère du chômage. La différence entre les deux régimes tient au fait que dans le chômage «classique », le marché des biens ne contribue pas à l'explication du chômage (i.e. même si les plans de production sont insuffisants pour satisfaire la demande, les entreprises ne subissent aucune contrainte sur le marché des biens), tandis que dans le chômage «keynésien », la demande de travail est contrainte par le marché des biens (i.e. la production des entreprises est bornée par une demande insuffisante); dans ce dernier cas, il s'ajoute une forme de chômage pouvant être rapprochée, dans un cadre de prix fixes à court terme, du chômage «temporaire » (Rueff) ou «conjoncturel » (Allais). Après avoir occupé pendant les
} 
Rueff et d'Allais, la différence majeure tient au fait que ces nouvelles théories constituent un étayage du caractère intrinsèquement non concurrentiel du marché du travail attribuable aux comportements même des agents. Dans le droit fil de ces approches, le modèle WS-PS («wage setting - price setting", Layard et Nickel (1985) ; Layard, Nickel et Jackman (1991)) explique le chômage par des rigidités attribuables à la fois aux comportements des agents et aux contraintes institutionnelles imposés par l'Etat. Proposé bien après l'équation d'Allais, ce modèle est fondé sur l'hypothèse réaliste de salariés et de firmes rationnels et négociant entre eux pour trouver un accord salarial. En explicitant les comportements au niveau microéconomique dans un cadre de concurrence imparfaite, le modèle WS-PS conduit à définir un taux de chômage dit «d'équilibre» pour lequel les aspirations des salariés et des entreprises sont compatibles, ce taux pouvant être à la fois plus élevé et plus fluctuant que le taux de chômage dit «naturel de plein emploi» à la Friedman. Layard-Nickel-Jackman (1991, p. 435) montrent que le modèle WS-PS rend assez bien compte des évolutions du taux de chômage dans 19 pays de l'OCDE dont la France: la spécification retenue explique en moyenne $86 \%$ de la variance des taux de chômage au cours de la période 1956-88. Ces résultats ont été corroborés avec diverses spécifications adaptées à la France (Bonnet et Mahfouz (1996), L'Horty et Sobczak (1997), L'Horty et Thibault (1998), Cotis et al. (1998), Chagny et al. (2002), l'Horty et Rault (2003), Gérard-Prenveille (2003), Heyer et al. (2005, 2007)). Dans l'ensemble, la littérature montre que le modèle WS-PS constitue un cadre d'analyse souple décrivant d'une manière réaliste les comportements des agents et conduisant à des résultats empiriques plutôt en faveur de cette approche.

Alors que Rueff est mort trop tôt pour pouvoir connaître la teneur des nombreuses contributions visées ci-dessus, tout se passe comme si Allais ne s'en était pas préoccupé, ce dernier n'ayant guère mentionné ces approches dans ses travaux postérieurs à 1980 (cf. notamment Allais (1999)) ; réciproquement, fondé sur le paradigme néo-keynésien, le modèle WS-PS ne se présentent bien sûr aucunement comme un héritage des travaux de ces deux auteurs. Néanmoins, en considérant une version simple et statique de ce modèle ${ }^{47}$, nous nous proposons de montrer que, sous certaines conditions, il est possible d'établir un lien formel entre le chômage d'équilibre déduit du modèle WS-PS et les approches de Rueff et d'Allais. Outre le fait que cet exercice permettra d'interpréter les contributions de ces deux auteurs avec des fondements microéconomiques explicites, il contribuera à éclairer les raisons du défaut de spécification que nous avons constaté à propos de l'équation d'Allais.

années 1970 une place importante dans l'analyse macroéconomique, la théorie des équilibres à prix fixes a pratiquement disparu des contributions scientifiques. L'absence d'une modélisation de la fixation des prix jointe au relâchement historique du contrôle administratif de ces derniers par les Etats - notamment en France avec l'abandon des prix administrés en 1984, alors même que les chercheurs français étaient situés au tout premier plan dans cet axe de recherche - sont sans doute les principaux facteurs permettant de comprendre l'abandon de ce programme (peut-être pas d'une manière définitive...).

${ }^{47}$ Les approches dynamiques du modèle WS-PS placent la négociation des salaires entre syndicats et employeurs dans un cadre intertemporel sur un horizon donné (voir tout particulièrement Cahuc et Zylberberg (1999) ainsi que d'Autume (2001)). Dans ce cadre théorique plus sophistiqué, il devient possible de distinguer une courbe WS de court terme et une courbe WS de long terme, et de déterminer à la fois les niveaux d'équilibre du chômage, du salaire réel et de l'intensité capitalistique; en outre, par rapport au modèle statique où le taux de marge est supposé exogène, ce taux dépend positivement du niveau d'emploi. Par rapport aux versions statiques, les versions dynamiques du modèle sont beaucoup plus complexes et nécessitent des hypothèses additionnelles sur l'horizon, les anticipations, le taux d'actualisation et le processus d'accumulation du capital. 
L'hypothèse d'une productivité marginale du travail décroissante jointe à celle d'entreprises maximisant leurs profits conduit ces dernières à fixer les prix en l'emploi de manière satisfaire l'expression suivante du salaire réel offert ${ }^{48}$ :

$$
\text { PS } \quad \frac{S_{t}^{p s}}{P_{t}}=\prod_{t} \phi_{1}\left(U_{t}, z_{t}^{p}\right)
$$

où $S_{t}^{p s}$ est le salaire offert par la firme représentative compte-tenu du niveau des prix $P_{t}$ qu'elle juge optimal de fixer, $\Pi_{t}$ la productivité du travail (supposée exogène), et $\phi_{1}$ une fonction dont la forme dépend de la fonction de production, et dont les arguments sont le taux de chômage $u_{t}$ et un ensemble d'autres variables $z_{t}^{p}$ conditionnant la fixation des prix par les entreprises (dont notamment le coin fiscalo-social et le taux de marge désiré qui sont supposés exogènes au modèle). L'influence positive du taux de chômage traduit ici le fait que, dans le cas général, l'efficacité du travail croît lorsque les effectifs diminuent, incitant ainsi la firme à offrir, toutes choses égales par ailleurs, un salaire réel plus élevé. Simultanément à ce comportement des firmes, l'objectif des syndicats est de maximiser le revenu des salariés compte-tenu de la probabilité pour ces derniers d'être au chômage et de recevoir dans ce cas un «équivalent revenu » égal aux allocations chômage augmentées de la désutilité du travail et de la valeur monétaire du travail domestique (d'Autume, 2001). On peut alors montrer qu'un chômeur n'acceptera de travailler que s'il touche un salaire au moins égal à cet «équivalent revenu» encore appelé «salaire de réserve». En effet, l'utilité marginale (consommation et temps de loisir) étant supposée décroissante, le programme du salarié représentatif conduit à la solution générale suivante dans laquelle le salaire de réserve apparaît comme argument du salaire désiré par les salariés :

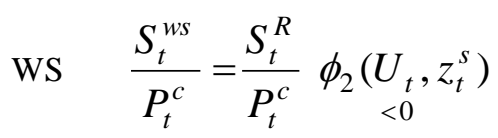

où $S_{t}^{w s}$ est le salaire désiré, $P_{t}^{c}$ le niveau des prix à la consommation considéré par les salariés pour évaluer leur pouvoir d'achat, $S_{t}^{R}$ leur salaire de réserve, $\phi_{2}$ une fonction dont la forme dépend de la fonction d'utilité, et $z_{t}^{s}$ un vecteur d'autres variables conditionnant les exigences salariales (parmi lesquelles le coin fiscalo-social). Dans ce cadre d'analyse simplifié, et selon le principe du «droit à gérer », les entreprises fixent leurs effectifs en fonction des accords salariaux réalisés avec les syndicats, le taux de chômage pouvant dès lors jouer le rôle de variable d'ajustement entre les revendications des deux parties, à condition que le marché du travail soit suffisamment flexible. ${ }^{49}$

En considérant classiquement les logarithmes des variables (voir, parmi bien d'autres, Bonnet (1999)), les équations WS et PS ci-dessus permettent d'inférer les équations (1) et (2) ci-dessous, où les variables en minuscules correspondent aux logarithmes des variables en majuscules, excepté pour les variables exprimées en taux. Comme cela est habituel, l'équation (1) est obtenue en considérant d'emblée que le taux de marge $m_{t}$ et le taux des cotisations sociales à la charge des entreprises $c e_{t}$ appartiennent au vecteur $z_{t}^{p}$ des variables

\footnotetext{
${ }^{48}$ Nous nous inspirons ici des présentations faites par Blanchard et Katz (1997) et Bonnet (1999).

${ }^{49}$ Par exemple, si le salaire réel désiré par les salariés est plus grand que celui proposé par les employeurs, des licenciements - et donc une augmentation du chômage - auront pour effet d'abaisser le premier et d'augmenter le second, conduisant ainsi à une valeur intermédiaire du salaire réel négocié.
} 
conditionnant la fixation des prix ; de même, le taux des cotisations à charge des salariés $c s_{t}$ appartient d'emblée au vecteur des variables $z_{t}^{s}$ conditionnant le salaire désiré avant impôt par les salariés (équation (2)). En outre, afin de rapporter le taux de salaire offert par les employeurs au même indice de prix que celui considéré par les salariés, l'équation PS a été multipliée à gauche et à droite par le rapport de prix $P_{t} / P_{t}^{c}$; cela signifie que, outre le taux de marge et le taux des cotisations, les firmes répercutent également sur leurs prix de vente l'écart entre ces derniers et les prix à la consommation, traduisant ainsi l'influence des termes de l'échange (prix des exportations / prix des importations) $:^{50}$

$$
\begin{aligned}
& \text { PS } \quad s_{t}^{p s}-p_{t}^{c}=\left(p_{t}-p_{t}^{c}\right)-c e_{t}+\pi_{t}+k^{p} U_{t}-m_{t}-f_{t}^{p} \quad k^{p} \geq 0 \\
& \text { avec }\left(p_{t}-p_{t}^{c}\right)=\tau_{t} \theta_{t} ; \tau_{t}: \text { taux d'ouverture et } \theta_{t}: \text { termes de l'échange } \\
& \text { WS } \quad s_{t}^{w s}-p_{t}^{c}=\left(s_{t}^{R}-p_{t}^{c}\right)+c s_{t}-k^{s} U_{t}+f_{t}^{s} \quad k^{s}>0 \\
& s_{t}^{p s} \text { : taux de salaire horaire brut offert par les entreprises } \\
& s_{t}^{\text {ws }} \text { : taux de salaire horaire brut désiré par les salariés (avant impôt) } \\
& s_{t}^{R} \text { : salaire de réserve (rémunération minimale nette exigée par les salariés) } \\
& p_{t}^{c} \text { : niveau des prix à la consommation (considéré par les salariés) } \\
& p_{t} \text { : niveau général des prix (fixé par les entreprises) } \\
& c e_{t} \text { : taux de prélèvement social supporté par les entreprises (par rapport au salaire brut) } \\
& c s_{t} \text { : taux de prélèvement social supporté par les salariés (par rapport au salaire net) } \\
& m_{t} \text { : taux de marge brut désiré et fixé par les entreprises } \\
& \pi_{t} \text { : productivité horaire du travail } \\
& U_{t} \text { : taux de chômage } \\
& k^{p} \text { : sensibilité au taux de chômage du niveau des prix fixé par les entreprises } \\
& k^{s} \text { : sensibilité au taux de chômage du salaire réel désiré par les salariés } \\
& f_{t}{ }^{p} \text { : autres facteurs conditionnant la fixation des prix } \\
& f_{t}^{s} \text { : autres facteurs conditionnant les exigences salariales }
\end{aligned}
$$

Le taux de chômage d'équilibre $U_{t}^{*}$ correspond à la valeur du taux de chômage telle les aspirations des deux camps sont compatibles, soit $s_{t}^{p s}=s_{t}^{w s}$, ce qui conduit à :

$$
\begin{aligned}
& U_{t}^{*}=\alpha_{o}\left[s_{t}^{R}+c s_{t}+c e_{t}-p_{t}-\pi_{t}+m_{t}+f_{t}\right] \\
& \text { avec } \alpha_{o}=1 /\left(k^{s}+k^{p}\right) \text { et } f_{t}=f_{t}^{s}+f_{t}^{p}
\end{aligned}
$$

La valeur du coefficient $\alpha_{o}=1 /\left(k_{s}+k_{p}\right)$ est d'autant plus petite que la somme des sensibilités $k_{s}$ et $k_{p}$ des salaires et des prix par rapport au sous-emploi est grande, ce qui signifie que $\alpha_{o}$ est d'autant plus petit que le chômage joue un rôle important en tant que

\footnotetext{
${ }^{50}$ Les équations (4) et (5) s'inspirent des présentations faites par Le Bihan et Sterdyniak (1998) et Simonnet (2008).
} 
variable d'ajustement entre les salaires et les prix au cours des négociations ; autrement dit, $\alpha_{o}$ est d'autant plus grand que le marché du travail est rigide. A ce propos, on relève que l'analyse de Rueff repose comme chez Keynes sur la considération de rigidités nominales, alors que le modèle WS-PS est directement fondé des rigidités réelles, comme c'est aussi le cas chez Allais. Cependant, cela n'interdit pas un rapprochement formel entre Rueff, Allais et WSPS. En effet, l'équation (6) montre que le chômage d'équilibre dépend de l'écart relatif entre le salaire réel de réservation augmenté des taux de cotisations sociales totales et la productivité du travail (soit $s_{t}^{R}+c s_{t}+c e_{t}-p_{t}-\pi_{t}$ ), ainsi que du taux de marge et de divers facteurs conjoncturels et structurels des salaires et des prix fixés par les entreprises, non explicités par les équations (2) et (3) mais dont l'existence est indiquée par la variable $f_{t}$. Par rapport à Rueff et l'Angleterre des années 1920, on voit que si (i) le salaire de réservation croit comme le salaire net moyen, soit $s_{t}=s_{t}^{R}+s_{o}\left(s_{o}>0\right)$ (ce qui était le cas à cette époque puisque la «dole » restait proche du salaire moyen), (ii) $c s_{t}=c s_{o}$ et $c e_{t}=c e_{o}$ (les taux des cotisations sociales sont constants, ce qui semble pouvoir être admis à l'époque), (iii) le taux de marge désiré par les entreprises est constant $\left(m_{t}=m_{o}\right)$, et enfin (iv) les facteurs «temporaires» et «minimum» supposés être représentés par $f_{t}$ ont une variabilité négligeable par rapport au chômage «permanent», on retrouve formellement la «loi de Rueff » reliant le taux de chômage à l'écart relatif entre le salaire réel et la productivité (voir figure 4).

Pour parvenir à l'équation du chômage (1) d'Allais, les conditions sont moins restrictives : il suffit d'admettre (i) et (iii), sachant que (iv) est assouplie dans la mesure où les «autres facteurs » «conjoncturels » et «technologiques» $f_{t}$ figurant dans l'équation (3) peuvent être résumés respectivement par la somme d'un élément proportionnel à la composante conjoncturelle de la production $\log \frac{Q_{t}}{Q_{T t}}$ et d'une constante $f_{o}$ traduisant l'influence de facteurs frictionnels, soit $f_{t}=b \log \frac{Q_{t}}{Q_{T t}}+f_{o} \quad\left(b>0 ; f_{o}>0\right)$. Dans le cadre WSPS, une interprétation envisageable de la présence de l'écart de production - lequel dépend bien sûr lui-même de variables $X_{i t}$ que cet écart est supposé résumer - est qu'il peut conditionner $f_{t}$ par le biais des anticipations de croissance. En effet, en raison de l'hypothèse de rendements décroissants, les agents négociateurs anticiperont en principe un taux de croissance de la production d'autant plus petit (grand) que la production se rapproche (s'écarte) de son maximum correspondant à l'utilisation de toutes les capacités de production ; dans ces conditions, une augmentation (baisse) de $\log \frac{Q_{t}}{Q_{T t}}$ traduira l'attente d'une croissance moins (plus) forte de la production conduisant les syndicats à des exigences salariales moins (plus) élevées et à un accueil moins bon (meilleur) de la part les employeurs, Ces comportements conduiront ainsi à des hausses simultanées de $f_{t}^{s}$ et $f_{t}^{p}$ donc de leur somme $f_{t}$, et in fine d'après (6), à une augmentation du chômage d'équilibre. Ces conditions reportées dans l'équation (6) conduisent à la valeur suivante du chômage d'équilibre :

$$
U_{t}^{*}=\alpha_{o}\left[\left(s_{t}+c s_{t}+c e_{t}-p_{t}\right)-\pi_{t}+b \log \frac{Q_{t}}{Q_{T t}}+u_{o}\right] \quad u_{o}=m_{o}+f_{o}-s_{o}>0
$$


On voit que d'après (7), le chômage d'équilibre peut être décomposé en trois éléments et se confond ainsi formellement avec l'équation (1) d'Allais : (a) un élément traduisant la répartition de la valeur ajoutée égal à l'écart relatif entre le salaire réel (augmenté des cotisations sociales) et la productivité du travail (i.e. la composante $\alpha_{o}\left[\left(s_{t}+c s_{t}+c e_{t}-p_{t}\right)-\pi_{t}\right]$ correspondant au chômage «chronique » $U_{1 t}$ d'Allais ou «permanent» de Rueff $^{51}$ ), (b) un élément proportionnel à l'écart de production (i.e. la composante $\alpha_{o} b_{o} \log \frac{Q_{t}}{Q_{T t}}$ correspondant au chômage «conjoncturel» $U_{2 t}$ d'Allais ou «temporaire » de Rueff), et (c) un élément $\alpha_{o} u_{o}$ traduisant les facteurs frictionnels (i.e. la composante «technologique » $U_{3 t}$ d'Allais ou «minimale » de Rueff).

Suivant cette spécification particulièrement simple du modèle WS-PS, on constate que le contenu du chômage d'équilibre a changé de nature par rapport aux conceptions de Rueff et d'Allais car ce contenu ne se limite plus au seul chômage «permanent » ou «chronique » mais fait aussi intervenir l'ensemble des facteurs conjoncturels ou frictionnels intervenant dans la détermination des salaires et des prix. Par ailleurs, la relation (7) suggère des raisons plausibles au défaut de spécification constaté avec l'équation d'Allais (voir Annexe 2). On peut notamment penser à la relation de proportionnalité entre le salaire observé et le salaire de réserve, à la constance du taux de marge, à la spécification des facteurs conjoncturels par l'écart de production, mais aussi à l'hypothèse implicite d'un degré de rigidité constant du marché du travail traduite par le coefficient $\alpha_{o}{ }^{52}$ On voit donc que le fait de regarder les approches de Rueff et d'Allais sous l'angle du modèle WS-PS illustre bien l'ampleur des progrès scientifiques accomplis.

\section{Conclusions}

Conformément aux idées défendues par l'école néoclassique, Rueff a montré empiriquement dans l'Angleterre des années 1920 qu'un niveau de salaire réel durablement supérieur à sa valeur d'équilibre concurrentiel génère du chômage «permanent ». Ce résultat statistique connu sous l'appellation de «loi de Rueff» constitue un apport original ayant marqué les esprits tant des économistes que des hommes politiques de l'époque. Même si Rueff avait remarquablement relié son analyse du chômage aux faits et institutions de l'Angleterre des années 1920, il est plus juste de faire ici état d'un résultat original que d'une pensée ou une démarche méthodologique qui soient à proprement parlé novatrice. Cependant, il était nécessaire de revisiter cette contribution pour en exhiber toute sa richesse, car la pensée de Rueff sur le chômage est plus large que celle focalisée sur le seul chômage «permanent», l'auteur envisageant aussi l'existence d'un chômage conjoncturel dit «temporaire » et d'un chômage frictionnel dit «minimal». Il était également nécessaire de compléter l'analyse empirique de Rueff, lequel admettait des hypothèses trop simplificatrices pouvant remettre en cause l'interprétation économique qu'il défendait. En premier lieu, Rueff supposait que la productivité du travail est constante, ce qui constitue une hypothèse forte sur

\footnotetext{
${ }^{51}$ A noter cependant une différence : Allais considérait la tendance de cette composante, alors qu'il s'agit ici de la valeur observée de cette dernière.

${ }^{52}$ Les analyses de long terme de Boyer (1978) et Villa (1994) montrent que la flexibilité du marché du travail est en France un phénomène variable et conditionnant la relation entre le salaire et le chômage. Voir encore Bonnet (1999) qui présente une analyse empirique de l'importance de la rigidité des salaires dans les pays de l'OCDE.
} 
la variable traduisant la valeur d'équilibre du salaire réel ; sur ce point, nous avons montré qu'en relâchant cette hypothèse, non seulement la «loi de Rueff » reste vérifiée, mais encore qu'elle l'est avec encore plus de précision. En second lieu, nous avons montré que la conjecture de Pigou suivant laquelle la baisse cyclique des prix aurait généré du chômage conjoncturel en raison d'une baisse de la production ne saurait expliquer l'ampleur du chômage anglais des années 1920; ceci étaye indirectement la thèse du chômage «permanent » en tant que facteur explicatif principal, et ceci même si nos résultats suggèrent que pendant les années 1919-21 et 1930, la baisse de la production a très vraisemblablement produit un chômage conjoncturel s'ajoutant au chômage permanent. Enfin, dans le but d'éclairer les débats interprétatifs entre Rueff et les économistes de son époque, nous avons présenté des tests économétriques simples dits de «causalité » qui tendent à confirmer l'hypothèse de Rueff suivant laquelle, au cours de la période 1919-30, les salaires réels (et non les salaires nominaux) conditionnent le chômage, et non l'inverse.

Dans le droit fil de ces résultats, cet article a montré que l'approche théorique d'Allais (1943) suivie beaucoup plus tard (en 1980) par son équation empirique du chômage - restée méconnue car non publiée dans un support académique mais dans un rapport au CNRS - se décalque sur les trois types de chômage évoqués par Rueff, Allais considérant à la fois un chômage «chronique » (i.e. «permanent»), un chômage « conjoncturel » (i.e. «temporaire ») et un chômage «technologique » (i.e. «minimal»). Allais montrait que, bien que très simple, son équation pouvait décrire la hausse du chômage français au cours des années 1952-1978, et nous avons montré que cette équation permet aussi de représenter les grands traits de l'évolution du chômage anglais des années 1920 et du chômage français des années 19702008 ; cependant, les propriétés statistiques indésirables des résidus indiquent sans ambiguïté pour ces trois échantillons que cette équation souffre d'un défaut de spécification.

Rueff peut être considéré comme un pionnier de la validation expérimentale de la vision (néo)classique du chômage, validation réalisée avant la publication de la «Théorie générale de l'emploi, de l'intérêt et de la monnaie » de Keynes (1936). La «loi de Rueff» a très vraisemblablement influencé la rédaction de la «Théorie Générale » d'après laquelle la rigidité à la baisse des salaires intervient dans la détermination de l' «équilibre de sousemploi ». Toutefois, la différence majeure entre Rueff-Allais et Keynes est que, même en l'absence d'indemnités versées aux chômeurs, ce dernier ne croyait pas à l'établissement d'un équilibre automatique du marché du travail, idée reprise avec le concept moderne de taux de chômage d'équilibre dont le modèle de concurrence imparfaite WS-PS de Layard-NickelJackman (1991) est très représentatif. A ce propos, nous avons vu que tant le chômage «permanent » de Rueff que le chômage «chronique »d'Allais représentent dans l'esprit de ces deux auteurs un chômage d'équilibre, alors que, sous certaines conditions, le modèle WSPS permet de définir une valeur d'équilibre du taux de chômage plus large pouvant être vue comme regroupant les trois types de chômage distingués par Rueff et Allais. Outre le fait que ce modèle présente l'avantage d'expliciter les comportements microéconomiques qui sont sous-jacents aux variables expliquant le chômage au niveau macroéconomique, il permet d'identifier des raisons plausibles au défaut de spécification de l'équation d'Allais, laquelle apparaît trop simple pour pouvoir traduire l'évolution du chômage d'une manière satisfaisante.

Au total, que la source de rigidité soit nominale ou réelle, les confrontations entre la contribution de Rueff et les approches ultérieures du chômage montrent d'une manière 
éclatante que «l'excès de salaire réel » est un concept central omniprésent. La pertinence théorique et empirique de ce phénomène a été établie par les modèles explicatifs du chômage qui se sont succédés au cours du temps, ceci que l'on regarde l'approche d'Allais, la théorie des équilibres à prix fixes, la nouvelle économie keynésienne du travail, ou encore le modèle WS-PS. Ce «fil rouge » semble très naturel, car le salaire réel détermine simultanément les conditions de vie des travailleurs et le coût de production des entreprises, ce coût devant à l'évidence être mis en rapport avec la productivité du travail.

\section{REFERENCES}

Allais M. (1943), Traité d'Economie Pure, Clément Juglar Ed, 1994, Paris, 912 p.

Allais M. (1971), «Salaires, prix, emploi », in Les conditions monétaires du développement économique, Fascicule III-5, Université de Paris Ouest, Faculté de Droit et des Sciences Economiques, année académique 1970-1971, 501-20

Allais M. (1980), «Les facteurs déterminants du chômage français, 1952-1978 », Rapport d“Activité Scientifique 1978-1980 (CNRS), Centre d'Analyse Economique, Septembre 46-67

Allais M. (1981), « Le chômage et les charges salariales globales », Le Monde, 14-15 juin

Allais M. (1999), La mondialisation, la destruction des emplois et de la croissance : l'évidence empirique, Clément Juglar Ed., seconde éd. 2007, Nancy

d'Autume A. (2001), "Le modèle WS-PS et le chômage d'équilibre », Cahiers de la MSE N83 (halshs-00452567), étude réalisée pour le compte de la Direction de la Prévision du Ministère de l'Economie, des Finances et de l'Industrie, Oct., 50 p.

Azariadis C. (1975), "Implicit contracts and underemployment equilibria", Journal of Political Economy, 83, 1183-1202

Béraud A. (2003), «Keynes et Pigou sur le salaire monétaire et l'emploi : une synthèse du débat », L'Actualité économique, Revue d'analyse économique, 79, 1-2, mars-juin 2003

Barro J. et H.I. Grossman (1971), « A General Disequilibrium Model of Income and Employment », American Economic Review, 61, 82-93

Basu S. et J.G. Fernald (2001), "Why is productivity procyclical? Why do we care"? in Hulten, Dean et Harper, New developments in productivity analysis, University of Chicago Press for the NBER

Benassy J.-P. (1975), « Neo-Keynesian Disequilibrium Theory in a Monetary Economy », Review of Economic Studies, 42, 503-23

Blanchard O.J. et Katz, L.F. (1999), "Wage Dynamic: Reconciling Theory and Evidence”, American Economic Review, 89(2), 69-74

Bonnet, X., Mahfouz S. (1996), The Influence of different Specifications of Wages-Prices Spirals on the Measure of the NAIRU: the Case of France, INSEE, Direction des Etudes et Synthèses Economiques, document de travail G9612

Bonnet X. (1999), «Peut-on mettre en évidence des rigidités à la baisse des salaires nominaux ? Une étude sur quelques grands pays de l'OCDE », Revue Economique, 50(3), 557-70

Boyer R. (1978), «Les salaires en longue période », Economie et statistique, 103, Sept., 27-57 
Broadberry S.N. (1997), The productivity race, British manufactoring in international perspective, 1850-1990, CUP, NY, 455 p.

Cahuc P. et A. Zylberberg (1999), «Le modèle WS-PS », Annales d'Economie et de Statistique, 53, 130

Clower R. W. (1965), «The Keynesian Counter-Revolution: a Theoretical Appraisal », In Hahn \& Brechling eds) The Theory of Interest Rates, Londres, Macmillan

Cotis J-P, R. Meary et N. Sobczak (1998), «Le chômage d'équilibre en France : une évaluation », Revue Economique, 49 (3), 921-35

Dos Santos Ferreira R. (1999), "La Relation Salaires-Emploi sous l'Eclairage de la Concurrence Imparfaite", Cahiers d'Economie Politique, 34, 15-40

Dreze J. H. (1975), «Existence of an Equilibrium under Price Rigidity and Quantity Rationing », International Economic Review, 301-320

Dunlop J. (1938), “The Movement of Real and Money Wages”, Economic Journal, 48(191), 413-34

Friedman M. A. Schwartz (1982), Monetary trends in the United States and the United kingdom - their relation to income, prices and interest rates, 1867-1975, NBER, The University of Chicago Press.

Gomez C. (2012), «Chômage et coûts salariaux : le retour de la « loi de Rueff - Rueff, Allais,... Artus », note de travail, version 1, http://osonsallais.wordpress.com/2012/11/17/c-gomez-chomage-etcouts-salariaux/

Heyer E et X.Timbeau (2002), «Le chômage structurel à 5\% en France ? », Revue de l'OFCE, 2002/1, $\mathrm{N}^{\circ} 80$

Heyer E., F. Reynes et H. Sterdyniak (2005), «Variables observables et inobservables dans la théorie du taux de chômage d'équilibre. Une comparaison France/Etats-Unis », Revue Economique, 56(3), 593-604

Heyer E, Reynes F et H. Sterdyniak (2007), "Structural and reduced approaches of the equilibrium rate of unemployment, a comparison between France and the United States", Economic Modelling, 24, 4265

Keynes J. M. (1936), The General Theory of Employment, Interest and Money, London: Macmillan reprinted (2007)

Keynes J. M. (1939), "Relative Movements of Real Wages and Output", The Economic Journal, 49(193), 34-51

Layard R. et S. Nickel (1985), «The cause of British unemployment», National Institute Economic Review, 111, 62-85

Layard R., S.Nickel et R. Jackman (1991), Unemployment, Oxford, Oxford University Press (reprinted 2009)

Le Bihan H. et H. Sterdyniak (1998), «Courbe de Phillips et modèle WS-PS, quelques remarques», Revue Economique, 49(1), 937-48

L'Horty Y. et C. Rault, "Les causes du chômage en France : Une ré-estimation du modèle ws-ps “, Revue Economique, 54, 2003/2, 271 - 94 
L'Horty Y. et N.Sobczak (1997), « Les déterminants du chômage d'équilibre : estimation d'un modèle WS-PS », Économie \& prévision,. $\mathrm{N}^{\circ} 127,1, .101-16$

L'Horty Y et F.Thibault, (1997), «Le NAIRU en France : les insuffisances d'une courbe de Phillips », Economie et Prévision, 127(11), 83-99

L'Horty Y. et F. Thibault, (1998), «Le chômage d'équilibre, de quoi parlons-nous ?», Revue Fançaise d'Economie , 13(4), 1998, 51-81

Lindbeck A et D. Snower (1989), The Insider-Outsider Theory of Employment and Unemployment, Cambridge, Mass., MIT Press

Lucas R. E. et L. Rapping (1969), "Real Wages, Employment, and Inflation”, Journal of Political Economy, vol. 77, 721-54

Lucas R. E. Jr. (1972), «Expectations and the Neutrality of Money », Journal of Economic Theory, vol. $4,103-24$

Malinvaud E. (1977), The Theory of Unemployment Reconsidered, New York, Basil Blackwell

Meade J.E. (1939), Economie politique et politique économique, Paris, Payot

Okun, A. (1962), "Potential GNP: Its measurement and significance", American Statistical Association, Proceedings of the Business and Economics Section, 98-103

Patinkin D. (1956), Money, Interest and Prices, Londres, Harper and Row

Pettinger T. (2012), "UK economy in the 1920s", October 16, Economics, http://www.economicshelp.org/blog/5948/economics/uk-economy-in-the-1920s/

Phillips A. W. (1958), "The relationship between unemployment and the rate of change of money wages in the UK, 1861-1957', Economica, Novembre, 283-99

Pigou A.C. (1927), "Wage policy and unemployment”, The Economic Journal, 37 (147), 355-68

Pissarides, C. [1990], Equilibrium Unemployment Theory, Ed Basil Blackwell, N-Y

Prat G. (2013), «Rueff et le chômage : quels héritages ?», IPAG working paper N²013-26 \& Document de travail EconomiX N²014-1, 37 p.

Robinson J. (1947), Essays in the theory of employment, Hyperion Press

Rueff J. (1925), "Les variations du chômage en Angleterre », Revue Politique et Parlementaire, 32, Déc., 425-37

Rueff J. (1931), «L'Assurance-Chômage : Cause du chômage permanent », Revue d'Économie Politique, 45, Mars-Avril, 211-51

Rueff J. (1947), «Les Erreurs de la théorie générale de Lord Keynes », Revue d'Économie Politique, 57, janvier-février, p. 5-33 ; version anglaise : «The Fallacies of Lord Keynes' General Theory », The Quarterly Journal of Economics, 1947, 61, mai, 343-67

Rueff J. (1951), « Nouvelles discussions sur le chômage, les salaires et les prix », Revue d'Économie Politique, 61, 761-91

Rueff J. (1976), «La fin de l'ère keynésienne », Le Monde, 19 et 20/21 février 1976 
Rueff J. (1977), De l'aube au crépuscule, Autobiographie, Plon, Paris, 1977, 443 p.

Shapiro C. et J. Stiglitz (1984), "Equilibrium unemployment as a worker discipline device", American Economic Review, 74 (3), 433-44

Simonnet J-P. (2008), "Chômage d'équilibre : deux approches théoriques, NAIRU et WS-PS", http://www.lyc-arsonval-brive.ac-limoges.fr/jp-simonnet/spip.php?article152

Stigler G. (1962), "The Intellectual and the Marketplace", Selected Papers, no. 3. Chicago: University of Chicago Graduate School of Business

Tarshis L. (1939), “Changes in Real and Money Wages”, Economic Journal, 49, 150-54

Teulon F. (2014), « Jacques Rueff et la réforme du système monétaire international », IPAG Business School, Working paper $\mathrm{N}^{\circ} 515,18 \mathrm{p}$.

Villa P. (1994), « Chômage et salaires en France sur longue période », CEPII, document de travail $\mathrm{N}^{\circ} 94-13,37 \mathrm{p}$.

Woltjer P. et H. de Jong (2010), « Output and productivity measurement in a US/UK Comparison, 1900-1950: reconciling sectoral benchmarks and time series", University of Groningen Faculty of Economics and Business, November 6, Paper prepared for the Maddison Memorial Conference http://www.ggdc.net/maddison/maddison-project/conferences/2010-

11/maddison_conference_nov2010_woltjer_dejong.pdf

\section{Annexe 1 - Angleterre, 1919-1930 : évaluation du chômage "permanent », du chômage «temporaire » et du chômage «minimal » de Rueff suivant l'équation d'Allais}

L'estimation de l'équation (1) d'Allais sur la période d'analyse de Rueff (1931) a été réalisée sur seulement 12 observations annuelles suivant la méthode de Newey-West qui est robuste à l'autocorrélation et à l'hétéroscédasticité des résidus. Compte-tenu du nombre très réduit d'observations, les résultats sont bien sûr hautement indicatifs et doivent être regardés avec la plus grande prudence. Les séries du taux de chômage $\left(U_{t} \%\right)$, du salaire moyen $\left(S_{t}\right)$ et des prix de gros $\left(P G_{t}\right)$ sont les moyennes annuelles des valeurs trimestrielles utilisées par Rueff. La série de production $\left(Q_{t}\right)$ correspond au revenu réel donné dans Friedman et Schwartz (1982), tandis que $y_{t}$ est le trend du rapport entre le salaire réel $S_{t} / P G_{t}$ et la productivité du travail $\Pi_{t}$, cette dernière ayant été évaluée comme indiqué dans le texte cidessus (cf. §1.31). En supposant qu'il n'existe pas de chômage chronique en 1919, les résultats obtenus sont donnés sur la Table 4 :

Table 4 - L’équation du chômage d'Allais, Angleterre 1919-1930 (*)

\begin{tabular}{lcccr}
\hline \hline \multicolumn{1}{c}{ Variable } & Coefficient & Std. Error & t-Statistic & Prob. \\
\hline \hline$\left(y_{t}-y_{1}\right) / y_{1}$ & 45.6 & 8.12 & 5.62 & 0.000 \\
$\log Q_{t} / Q_{T t}$ & -45.7 & 15.89 & -2.88 & 0.018 \\
$C_{0}$ & 4.50 & 1.25 & 3.60 & 0.006 \\
\hline \hline R-squared & 0.765 & Mean dependent var & 10.46 \\
Adjusted R-squared & 0.713 & S.D. dependent var & 4.53
\end{tabular}


$\left({ }^{\star}\right)$ Equation estimée : $U_{t}=\underset{>0}{A} \frac{y_{t}-y_{1}}{y_{1}}+\underset{<0}{B} \log \frac{Q_{t}}{Q_{T t}}+C_{0}+\varepsilon_{t}$ avec :

$$
\begin{aligned}
& y_{t}: \text { trend HP de }=\left(S_{t} / P G_{t}\right) / \Pi_{t} ; y_{1} \text { : valeur de } y_{t} \text { en } 1919 \\
& \ln Q_{T t}: \text { trend } H P \text { (Hodrick-Prescott Filter) de } \ln Q_{t}
\end{aligned}
$$

On constate que les coefficients $A$ et $B$ sont significatifs avec les signes attendus ; on peut noter que, sous la condition $b=0$, la constante $C_{0}=4.5 \%$ représente la composante minimale (frictionnelle ou technologique) estimée du taux de chômage. La composante «chronique » («permanente») du chômage apparaît comme le principal facteur, mais les composantes «conjoncturelle » et frictionnelle expliquent aussi une partie du chômage. En fait, sur la période 1924-1930, la composante conjoncturelle prend des valeurs positives ou nulles, ce qui implique que l'évolution de la production a eu une influence négative sur le chômage : le chômage aurait été plus important sans cette composante ; par contre, sur la période 1921-24, cette composante conjoncturelle a des valeurs négatives, ce qui signifie qu'elle s'est ajoutée au chômage «permanent » pour rendre compte du chômage observé.

\section{Annexe 2 - L'équation d'Allais, France : 1970-2008}

Nous avons retenu des indicateurs statistiques différents de ceux utilisés par Allais car l'INSEE a publié depuis des statistiques plus générales sur les salaires et les taux de cotisation associés. Alors qu'Allais considérait le salaire dans les industries mécaniques et électriques, nous considérons le salaire net moyen de l'ensemble du secteur privé et semi-public. Alors qu'Allais considérait l'écart à la tendance linéaire de la production industrielle, nous considérons l'écart à la tendance $H P$ du PIB en volume. Par ailleurs, Allais considérait l'indice déflateur du PIB alors que, conformément au choix de Rueff, nous considérons l'indice des prix de gros. Alors qu'Allais utilisait un indice de productivité nette du travail, nous utilisons un indice brut, lequel minimise en principe l'importance de l'écart entre le salaire réel et la productivité. Enfin, pour estimer la variable $y_{t}$ nous avons retenu un filtre $H P$ alors qu'Allais considérait une moyenne mobile sur 5 ans. L'objectif étant la mise en évidence d'une relation structurelle, nous avons préféré exclure les années de turbulence correspondant à la crise financière mondiale ayant débuté à la mi-2007 (bien que relayée par une seconde phase de faillites bancaires à l'automne 2008, on peut considérer que les effets macroéconomiques n'ont pris une ampleur significative en France qu'en 2009).

Les données sont les suivantes :

$U_{t}$ : taux de chômage au sens du BIT à l'instant $t(\%)$

$y_{t}::$ trend du rapport entre le coût horaire réel total du travail (charges sociales incluses) et la productivité horaire du travail (filtre $H P$ ). Hypothèse de calage: absence de chômage chronique entre $1960\left(y_{1}=y_{1960}=1.123\right)$

$S_{t}:$ salaire moyen horaire net, base $1950=1$

$P G_{t}$ : indice général des prix de gros, base 1950=1 
$\Pi_{t}:$ indice de la productivité horaire du travail, base $1950=1$

$Q_{t}:$ PIB réel $; \ln Q_{T t}:$ filtre $H P$ sur $\ln Q_{t}$

L'équation (1) a été estimée avec la méthode de Newey-West. Les résultats obtenus sont présentés sur la table 5 :

Table 5 - L'équation du chômage d'Allais, France, 1970 - 2008

\begin{tabular}{|c|c|c|c|c|}
\hline Variable & Coefficient & Std. Error & t-Statistic & Prob \\
\hline$\left(y_{t}-y_{1}\right) / y_{1}$ & 21.6 & 3.42 & 6.31 & 0.000 \\
\hline $\log Q_{t} / Q_{T t}$ & -47.8 & 15.19 & -3.15 & 0.003 \\
\hline$C_{0}$ & 1.34 & 0.98 & 1.38 & 0.180 \\
\hline $\mathrm{R} 2$ & 0.889 & \multirow{2}{*}{\multicolumn{2}{|c|}{$\begin{array}{l}\text { Mean dependent var } \\
\text { S.D. dependent var }\end{array}$}} & 8.04 \\
\hline Adjusted R2 & 0.882 & & & 2.92 \\
\hline S.E. of regression & 0.998 & \multirow{2}{*}{\multicolumn{2}{|c|}{ Durbin-Watson stat }} & \\
\hline F-statistic & 144.2 & & & 0.43 \\
\hline
\end{tabular}

$\left.{ }^{*}\right)$ Equation estimée : $U_{t}=\underset{>0}{A} \frac{y_{t}-y_{1}}{y_{1}}+\underset{<0}{B} \log \frac{Q_{t}}{Q_{T t}}+C_{0}+\varepsilon_{t}$

avec :

$y_{t}:$ trend $\mathrm{HP} \mathrm{de}=\left(S_{t} / P G_{t}\right) / \Pi_{t} ; y_{1}:$ valeur de $y_{t}$ en 1960

$\ln Q_{T t}:$ trend $H P$ de $\log \log Q_{t}$

Les résultats de la table 4 indiquent que les composantes chronique et conjoncturelle du chômage ont les signes attendus; la non significativité de la constante $C_{0}$ incluant du chômage «technologique» pourrait être dû à l'hypothèse de calage de $y_{t}$ (supposant l'absence de chômage «chronique » en 1960) et/ou à une valeur implicite négative du paramètre $b$ intervenant dans la masure de l'excès de salaire réel. La figure 8 montre que les valeurs calculées d'après cette équation rendent compte du mouvement général du taux de chômage sur la période, l'écart entre le salaire réel et la productivité représentant la tendance en hausse du taux de chômage. Autrement dit, cette équation montre que la "loi de Rueff» peut être regardée comme sous-jacente à la dynamique du chômage français au cours de ces années ; un test $A D F$ (Augmented Dickey-Fuller) approprié (non reproduit) a montré que l'on peut admettre l'hypothèse de stationnarité des résidus au seuil de $5 \%$, ce qui valide l'hypothèse d'une relation de long terme. Cependant, on constate des écarts très importants et durables entre les valeurs observées et calculées, les résidus étant à la fois significativement auto-corrélés (voir la statistique $D W$ ) et hétéroscédastiques (tests Arch non reproduits), ces résultats indiquant un défaut de spécification: même si elle tend grosso-modo à corroborer les idées d'Allais, l'évidence montre que cette équation est beaucoup trop simple pour rendre compte de l'évolution du chômage d'une manière satisfaisante. Le choix des indicateurs statistiques conditionne bien sûr les résultats ; à ce propos, notons que le remplacement des prix de gros par le déflateur du PIB ne permet guère d'obtenir des résidus ayant des propriétés satisfaisantes. 
Figure 8 - France, 1970 - 2008 : valeur observée du taux de chômage et valeurs calucllées suivant l'équation d'Allais

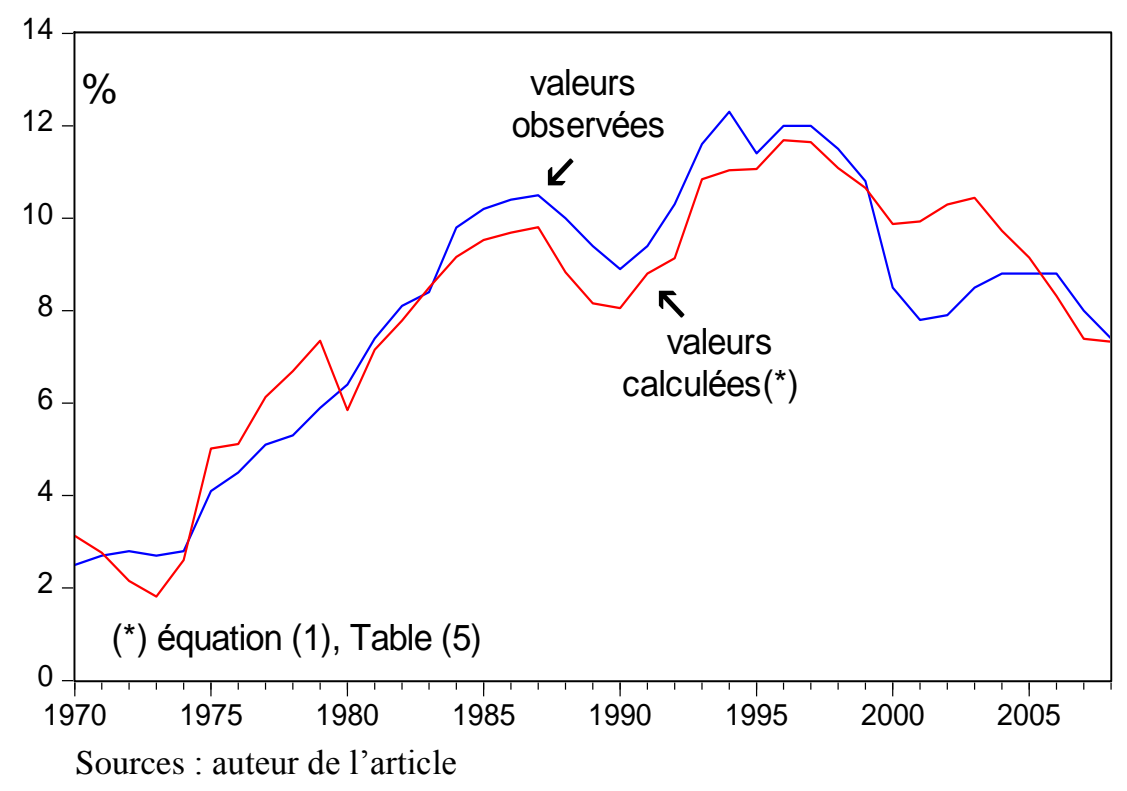

